\title{
Anxiety- and Depression-Like States Lead to Pronounced Olfactory Deficits and Impaired Adult Neurogenesis in Mice
}

\author{
Eleni Siopi, ${ }^{1,2}$ Marie Denizet, ${ }^{1,2}$ Marie-Madeleine Gabellec, ${ }^{1,2}$ Fabrice de Chaumont, ${ }^{3,4}$ Jean-Christophe Olivo-Marin, ${ }^{3,4}$ \\ Jean-Philippe Guilloux, ${ }^{5}$ Pierre-Marie Lledo, ${ }^{1,2 *}$ and Françoise Lazarini ${ }^{1,2 *}$ \\ ${ }^{1}$ Institut Pasteur, Unité Perception et Mémoire, F-75015 Paris, France, ${ }^{2}$ Centre National de la Recherche Scientifique, Unité Mixte de Recherche 3571 , F- \\ 75015 Paris, France, ${ }^{3}$ Institut Pasteur, Unité d'Analyse d'Images Quantitative, F-75015 Paris, France, ${ }^{4}$ Centre National de la Recherche Scientifique, Unité \\ de Recherche Associée 2582, F-75015 Paris, France, and ${ }^{5}$ Institut National de la Santé et de la Recherche Médicale UMR-S 1178, Faculté de Pharmacie, \\ Université Paris-Sud, Châtenay-Malabry F-92296, France
}

Numerous clinical reports underscore the frequency of olfactory impairments in patients suffering from major depressive disorders (MDDs), yet the underlying physiopathological mechanisms remain poorly understood. We hypothesized that one key link between olfactory deficits and MDD lies in hypercortisolemia, a cardinal symptom of MDD. Corticosterone (CORT) is known to negatively correlate with hippocampal neurogenesis, yet its effects on olfactory neurogenesis and olfaction remain unknown. Here we used a rodent model of anxiety/depression-like states, which is based on chronic CORT administration and studied the effects of the antidepressant fluoxetine (FLX) on behavior, olfaction, and adult neurogenesis in the dentate gyrus (DG), olfactory bulb (OB), and the olfactory epithelium (OE). Chronic CORT had no effect on cell proliferation in the OE or on olfactory sensory neurons projecting to the OB, but induced pronounced deficits in olfactory acuity, fine discrimination of odorants and olfactory memory. These alterations were accompanied by a significant decrease in the number of adult-born neurons in both the DG and OB. Remarkably, FLX not only reversed depression-like states as expected, but also improved olfactory acuity, memory, and restored impaired adult neurogenesis. However, fine olfactory discrimination was not restored. Morphological analysis of adult-born neurons in both the DG and the OB showed that dendritic complexity was not significantly affected by CORT, but was increased by FLX. These findings demonstrate an essential role for glucocorticoids in triggering olfactory impairments in MDD and highlight a novel therapeutic effect of FLX.

Key words: corticosterone; dentate gyrus; olfactory bulb; olfactory epithelium; serotonin; subventricular zone

Significance Statement

Increasing clinical reports show that major depression is characterized by pronounced olfactory deficits, yet the underlying mechanisms remain unknown. In this work, we used an endocrine model of depression to study whether hypothalamic-pituitaryadrenal axis perturbation could be sufficient to provoke olfactory impairments. We found that chronic corticosterone not only induces marked deficits in olfactory acuity, fine discrimination and olfactory memory, but also significantly decreases bulbar and hippocampal neurogenesis. Importantly, the antidepressant fluoxetine restores both adult neurogenesis and depressive states, and improves most olfactory functions. Our data reveal that impairment of hypothalamic-pituitary-adrenal axis during depression can lead to olfactory deficits and that the neurogenic effects of selective serotonin reuptake inhibitor antidepressants can successfully restore certain olfactory functions.

\section{Introduction}

Major depressive disorders (MDDs) are highly prevalent and debilitating conditions, whose pathophysiological mechanisms and

Received July 27, 2015; revised Nov. 12, 2015; accepted Nov. 30, 2015.

Author contributions: E.S., P.-M.L., and F.L. designed research; E.S., M.D., M.-M.G., and F.L. performed research; F.D.C., J.-C.O.-M., and J.-P.G. contributed unpublished reagents/analytic tools; E.S., M.D., M.-M.G., and F.L. analyzed data; E.S., P.-M.L., and F.L. wrote the paper.

This work was supported by the Agence Nationale de la Recherche "ANR-SAMENTA 2012," the "Laboratory for Excellence" programs "Revive" and "BioPsy," as well as the life insurance company "AG2R."; E.S. is supported by a postdoctoral grant from ANR-SAMENTA; and the Imagopole-Plateforme d'Imagerie Dynamique France bioimaging response to treatments remain poorly understood (Krishnan and Nestler, 2008). Increasing clinical reports underscore the frequency of olfactory impairments in patients suffering from MDDs (Pause et al., 2001; Negoias et al., 2010), yet, it remains infrastructure, supported by the French National Research Agency (ANR 10-INSB-04-01, Investments for the Future), for advice and access to the CV1000 system. We thank Raphaël Servignat d'Août for his contribution in the behavioral assays, Gabriel Lepousez for his technical help in olfactory epithelium analysis, and Denis David, Alain Gardier, and Emmanuelle Corruble for fruitfull comments on this project. Pierre-Marie Lledo's laboratory is part of the École des Neurosciences de Paris lle-de-France network. 
unclear how mood states and olfactory processes interact in psychiatric disorders such as MDDs.

Olfactory deficits in MDDs could be partly due to endocrine dysfunction, which is suspected to play a key role in anxiety/ depressive disorders. In fact, $\sim 80 \%$ of severely depressed patients exhibit chronic hyperactivity of the hypothalamic-pituitaryadrenal (HPA) axis, mainly reflected by an excess of plasma cortisol (Anacker et al., 2011). This phenomenon is modeled in rodents, which show HPA axis abnormalities and increased corticosterone (CORT) levels upon unpredictable chronic mild stress, early life stress, or chronic social stress (Lehmann et al., 2013). Both the hippocampus and the olfactory bulb (OB) are densely populated with receptors for glucocorticoids (Morimoto et al., 1996). Although the effects of glucocorticoids in the adult $\mathrm{OB}$ remain relatively unknown, they play an important role in the hippocampus by negatively regulating the HPA axis, resulting in stress resilience. In conditions of chronic stress, when there is a sustained overproduction of glucocorticoids, this inhibitory feedback is impaired, creating a cascade of hippocampal damage and resulting in maladaptive stress responses (Selye, 1950; Mirescu and Gould, 2006).

Abundant evidence suggests that chronic stress is highly regulated by adult hippocampal neurogenesis (Dranovsky and Hen, 2006). In rodents, adult neurogenesis is a key mechanism to maintain plasticity in response to both internal and environmental challenges (Lledo et al., 2006), and its functional relevance ranges from perceptive to cognitive functions, including mood regulation, pattern separation, learning, memory, and behavioral responses to olfactory cues (Breton-Provencher et al., 2009; Lazarini and Lledo, 2011; Alonso et al., 2012). Abnormal hippocampal neurogenesis is likely to participate in psychiatric disorders, such as depression (Sahay and Hen, 2007), and the production of adult-born hippocampal neurons is influenced by stress and glucocorticoids (Murray et al., 2008; David et al., 2009; Snyder et al., 2011). In MDD patients and mouse models of chronic stress, there is evidence for reduced hippocampal volume and impaired adult neurogenesis, both of which can be restored by antidepressant treatments (Campbell et al., 2004; Boldrini et al., 2009). Moreover, adult hippocampal neurogenesis is required for the response to antidepressants (Santarelli et al., 2003; David et al., 2009), and adult-born neurons are essential in restoring hippocampal inhibitory control over the HPA axis and in normalizing glucocorticoid levels following chronic stress (Snyder et al., 2011).

In this work, we determine whether such a link could be also drawn between HPA axis dysfunction and olfactory neurogenesis. In rodents, newborn neurons from the lateral wall of the subventricular zone (SVZ) migrate en route to the OB, where they differentiate into bulbar interneurons. The few existing studies on the impact of chronic stress on olfactory neurogenesis show mixed results. While prolonged mild stress or CORT treatment has been shown to decrease cell proliferation in the SVZ (Hitoshi et al., 2007; Lau et al., 2011), there is contradictory evidence that the latter is not sensitive to repeated stress (Chen et al., 2006; Surget et al., 2008). Nevertheless, clinical studies have reported that adults with a history of childhood stress or MDD can have

The authors declare no competing financial interests.

* P.-M L. and F.L. contributed equally to this work.

Correspondence should be addressed to either Dr Pierre-Marie Lledo or Dr Françoise Lazarini, Perception and Memory Laboratory, Institut Pasteur, 25 rue du Dr. Roux, F-75724 Paris Cedex 15, France, E-mail: pmlledo@pasteur.fr or lazarini@pasteur.fr.

DOI:10.1523/JNEUROSCI.2817-15.2016

Copyright $\odot 2016$ the authors $\quad 0270-6474 / 16 / 360519-14 \$ 15.00 / 0$ reduced OB volume (Negoias et al., 2010; Croy et al., 2013), suggesting a potential impact of chronic stress on $\mathrm{OB}$ functioning.

To investigate whether defects in the HPA axis could alter olfactory neurogenesis and olfaction, we used a neuroendocrine mouse model of anxiety/depression (David et al., 2009). We found that chronic CORT affects adult neurogenesis and induces both anxiety/depression-like states and olfactory impairments. We then report that coadministration of the antidepressant fluoxetine (FLX), a selective serotonin reuptake inhibitor, restores adult neurogenesis and attenuates the olfactory deficits.

\section{Materials and Methods}

Subjects

Experiments were performed using adult male C57BL/6Ntac mice (8 weeks old, 20-25 g), purchased from Taconic Farms. They were housed in groups of five and maintained in standard conditions (controlled room temperature and humidity, $12 \mathrm{~h}$ light/dark cycle, with lights on at 8:00 A.M., ad libitum access to dry food pellets and water) at the Pasteur Institute animal care facility, officially registered for experimental studies on rodents (approval number for animal care facilities: A 75-15-01-6 2014-720). These studies were performed in compliance with the French legislation (Decree of Feb 1, 2013, 2013-118) and the European Communities Council Directive of Sept 22, 2010 (2010/63/EEC). Experimental designs and procedures were approved by the local ethics committee (no. 2011-0020, CETEA 59 and no. 2013-0081, CETEA 89).

\section{Drugs}

The dose of CORT and FLX treatments was selected upon previous studies (David et al., 2009). CORT (4-pregnen-11b-diol-3 20-dione 21hemisuccinate, from Sigma-Aldrich) was dissolved in its vehicle $(0.45 \%$ hydroxypropyl- $\beta$-cyclodextrin, $\beta$-CD, Sigma-Aldrich) and was available ad libitum in drinking water $(35 \mu \mathrm{g} / \mathrm{ml} / \mathrm{d}$, equivalent to $\sim 5 \mathrm{mg} / \mathrm{kg} / \mathrm{d})$. FLX (Anawa Trading, $18 \mathrm{mg} / \mathrm{kg} / \mathrm{d}$ ) was delivered either with the vehicle or with CORT. During the period of water restriction, CORT was administered intraperitoneally (i.p.) as previously described (Harris et al., 2011).

\section{Design and treatment groups}

Mice were divided in a total of 11 treatment groups (total $n=105$ ) for four separate experimental sets.

Experimental set 1. Impact of CORT and FLX on behavior, olfaction and adult neurogenesis (Figs. 1, 2, 4-6). One group received the vehicle $(n=12)$ for 20 weeks and the second group received CORT $(n=20)$ for 20 weeks. The third group received CORT during 6 weeks and then CORT + FLX during 14 weeks $(n=35)$. Using standard behavioral tests, we confirmed the anxious-depressive phenotype of mice at 4 weeks of CORT treatment (Fig. 1). One test per day was performed in the following order: open field, elevated plus maze, novelty suppressed feeding and splash test. Then, olfactory tests were conducted at the eighth week of CORT treatment, using fewer mice in each group (vehicle group: $n=8$; CORT group: $n=8$; CORT +FLX group: $n=35$ ). The choice of mice was random and the remaining animals were eliminated from the study. Following 1 week of training in the olfactometers, olfactory tests lasted 5 weeks (Fig. 3). Olfactory perception was assessed 4 weeks following the onset of FLX administration. Simple and difficult olfactory discrimination (carvones and carvone mixtures) was assessed at 6 and 7 weeks, respectively, following the onset of FLX treatment. Finally, olfactory memory was assessed at 12 weeks following the onset of FLX treatment. All mice $(n=51)$ were subjected to behavioral essays at week 16 of CORT onset ( 1 test per day, as follows: open field, elevated plus maze, novelty suppressed feeding, splash test, tail suspension test, and forced swim test; Fig. 2) and to an olfactory memory test at week 18 (Fig. 3F). Immunohistochemistry was performed at week 20 using 10 mice from the CORT+FLX group, five mice from the vehicle group and five mice from the CORT-treated group, that were randomly chosen (see Figs. 5, 6).

Experimental set 2. Impact of chronic FLX on behavior (Fig. 3). One group received the vehicle $(n=10)$ and the other group received FLX 
A

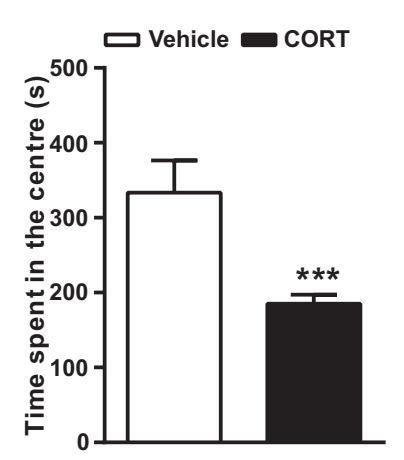

D Novelty suppressed feeding

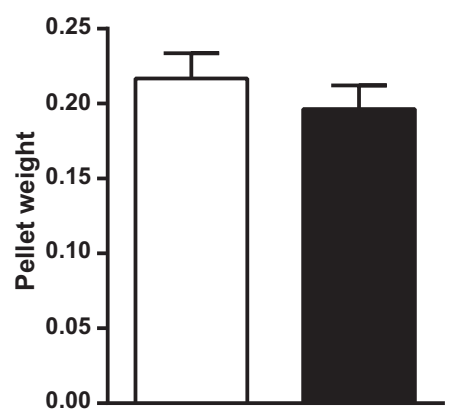

B

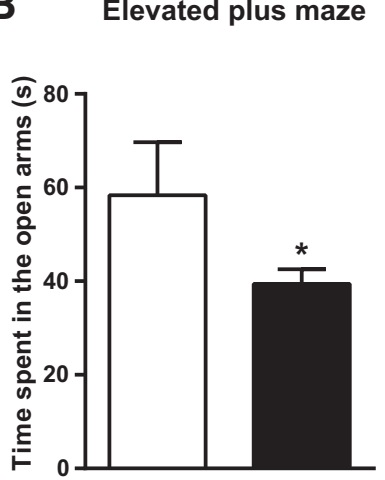

E

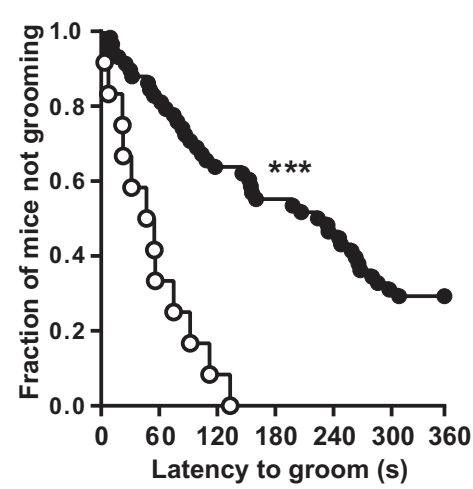

C Novelty suppressed feeding

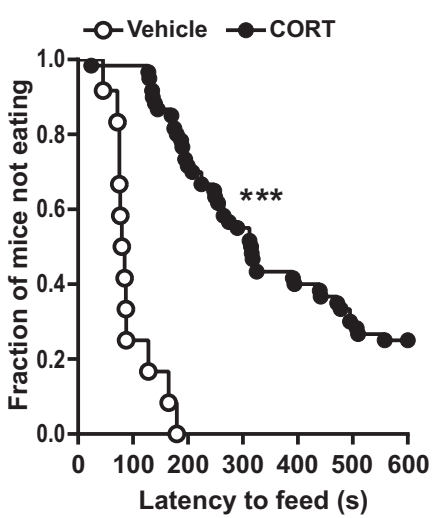

$\mathbf{F}$

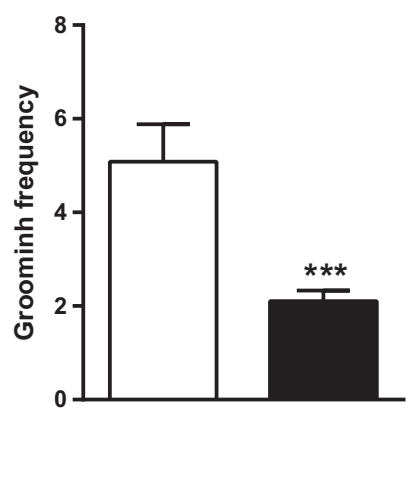

Figure 1. Chronic CORT treatment induces anxiety and depression-like states. Following 4 weeks of CORT administration, anxiety, and depression-like behaviors were assessed using the open field, elevated plus maze, novelty suppressed feeding test, and splash test. CORT decreased the time spent $(\boldsymbol{A})$ in the center of the open field and ( $\boldsymbol{B})$ in the open arms of the elevated plus maze. $\boldsymbol{C}$, Graph showing the latency to feed in the novelty suppressed feeding paradigm, presented as the fraction of mice that did not eat over a period of $10 \mathrm{~min}$. $\boldsymbol{D}$, CORT did not affect feeding drive. $\boldsymbol{E}$, CORT-treated mice displayed an increased latency to groom in the splash test. $\boldsymbol{F}$, The total number of grooming sessions within the 6 min trial was significantly decreased by CORT. Values are plotted as mean \pm SEM ( $n=12$ for vehicle-treated and $n=55$ for the CORT group). ${ }^{*} p<0.05$, ${ }^{* * *} p<0.001$ compared with vehicle-treated.

$(n=10)$ for 14 weeks (Fig. 3A). Olfactometry was performed following 2 weeks of FLX treatment and lasted 6 weeks. Mice were then subjected to behavioral analysis at week 10 ( 1 test per day as follows: open field, elevated plus maze, novelty suppressed feeding, splash test, tail suspension test and forced swim test) and to an olfactory memory test at week 12 .

Experimental set 3. Impact of CORT on cell proliferation in the olfactory epithelium (see Fig. 7). Immunohistochemistry was done using two groups of mice, one group that was treated with CORT during 4 weeks $(n=5)$ and a group that received the vehicle $(n=5)$.

Experimental set 4. Impact of FLX on the morphology of adult-born OB neurons in CORT-treated and naive mice (see Fig. 8). The first group $(n=4)$ received the vehicle and the second group $(n=4)$ received CORT during 11 weeks. The third group received CORT during 4 weeks and then CORT+FLX during 7 weeks $(n=4)$. The fourth group $(n=4)$ received the vehicle during 4 weeks and then FLX during 7 weeks (see Fig. $8 A$ ). A lentiviral vector was injected in the SVZ at week 8 to label adult newborn neurons (see Fig. $8 C$ ). Immunohistochemistry was performed at week 11 .

\section{Behavioral assessment}

A battery of behavioral tests was used for the mice included in experimental sets 1 and 2, to assess locomotor activity, anxiety and depressionlike behavior and olfaction.

Open-field. Animals were placed in white Plexiglas containers $(43 \times 43$ $\mathrm{cm}^{2}$ ) and their behavior was recorded by a video camera during $30 \mathrm{~min}$. A tracking system (Noldus Ethovision 3.0) was used to map center and periphery zones and to calculate the time spent in each zone.

Elevated plus maze. The test was conducted using a plus-cross-shaped apparatus made of black Plexiglas that was elevated $58 \mathrm{~cm}$ above the floor and comprised two open and two closed arms $(30 \times 6 \mathrm{~cm})$ that extended from a central platform $(7 \times 7 \mathrm{~cm})$. The Noldus Ethovision 3.0 tracking system was used to record behavior for $6 \mathrm{~min}$. The time spent and entries in center, open arm, and closed arm zones were calculated.

Novelty suppressed feeding. The test container used was a white plastic box $(50 \times 50 \times 20 \mathrm{~cm})$ whose floor was covered by wooden bedding. Twenty-four hours prior testing, all food was removed from the home cage. A single food pellet (regular chow) was adhered on a piece of Whatman paper and positioned in the center of the container that was brightly illuminated. The latency to eat was measured during $10 \mathrm{~min}$ as previously described (David et al., 2009). At the end of each session, the animals were immediately transferred into their home cage and the amount of food consumed over the subsequent 5 min period, as well as their body weight, were measured as a control of feeding drive.

Splash test. The test consisted in squirting $\sim 200 \mu \mathrm{l}$ of a sucrose solution on the dorsal coat of the mouse, as previously described (Surget et al., 2008). Grooming latency, frequency, and duration were assessed during 6 min.

Tail suspension test. Mice were suspended by the tail using adhesive tape on a metal rod of an automated tail suspension apparatus (Bioseb), as previously described (Cryan et al., 2005). Immobility time and power of movement were recorded during a 6 min period.

Forced swim test. The test was performed using an automated forced swim test software (Bioseb), as previously described (Garza et al., 2012). Total immobility time, swimming, and climbing states were measured during a 6 min session.

\section{Olfactometry}

Mice were partially water-deprived for 1 week and were then trained using an operant conditioning go/no-go procedure in computercontrolled olfactometers (Fig. $4 A$ ) as described previously (Alonso et al., 
A

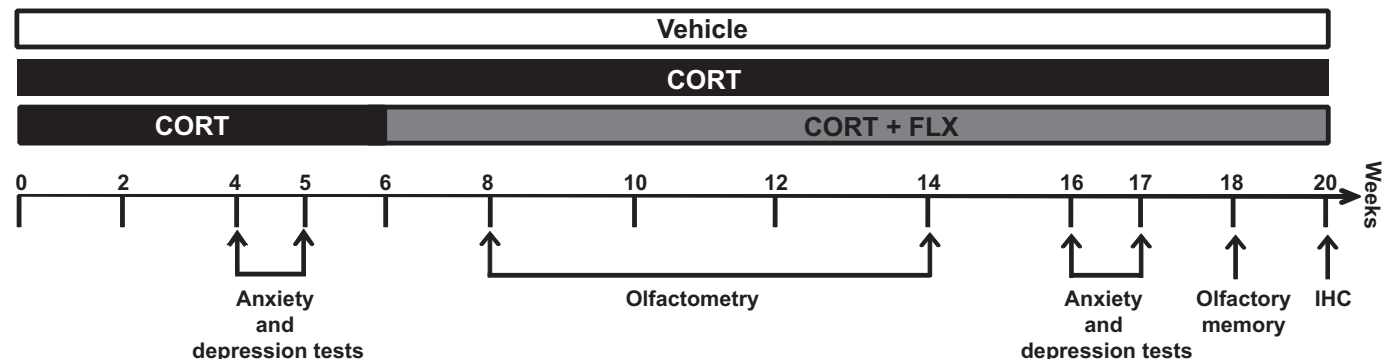

depression tests

B Novelty suppressed feeding

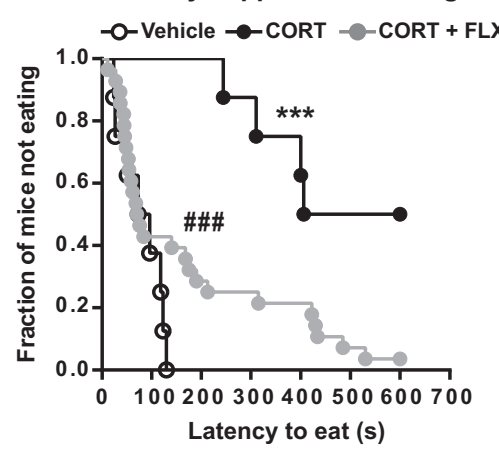

E

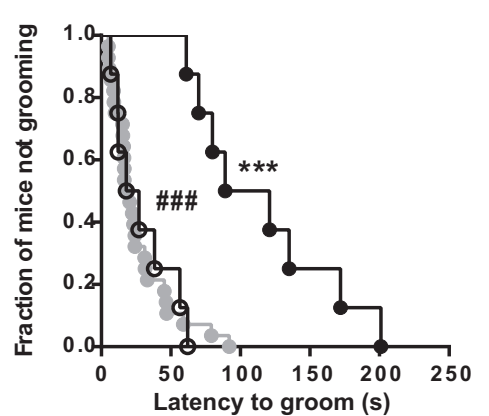

C Novelty suppressed feeding $\square$ Vehicle $\square$ CORT $\square$ CORT + FLX

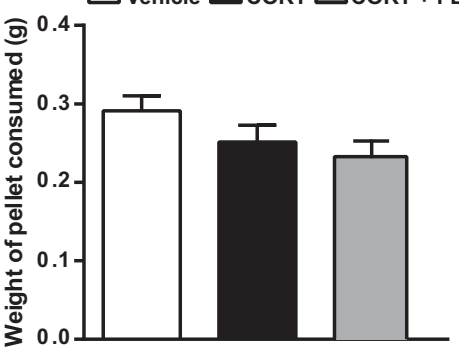

D Splash test

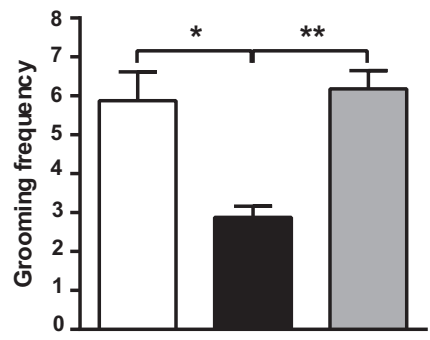

$\mathbf{F}$

Forced swim test

G

Tail suspension test

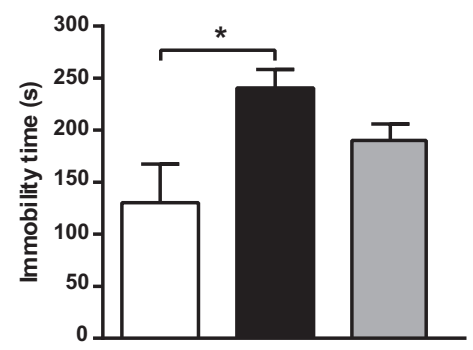

Figure 2. Effects of FLX on CORT-induced anxiety- and depression-like states. $A$, Diagram depicting experimental groups and study design. Animals were tested in the open field, elevated plus maze, novelty suppressed feeding, splash test, tail suspension test, and forced swim test paradigms successively, with a $1 \mathrm{~d}$ interval. $\boldsymbol{B}-\boldsymbol{G}$, Following 16 weeks of CORT and 10 weeks of CORT + FLX treatment, $(\boldsymbol{B})$ the latency to eat in the novelty suppressed feeding paradigm was significantly increased by CORT and attenuated by FLX. C, Neither CORT nor CORT + FLX induced any change in feeding drive, reflected by the amount of pellet consumed during $5 \mathrm{~min}$ after the test. $D, E, C O R T$-treated mice displayed both increased grooming latency and decreased grooming frequency in the splash test, which were abrogated by FLX.F,G, Average immobility time was not significantly affected by CORT in the tail suspension test, but it was notably increased in the forced swim test. FLX did not induce any statistically significant effect. Values are plotted as mean $\pm \operatorname{SEM}\left(n=8\right.$ for the vehicle and CORT groups, $n=35$ for the CORT $+{ }_{F L X}$ group $) .{ }^{*} p<0.05$, ${ }^{* *} p<0.01,{ }^{* * *} p<$ $0.001,{ }^{\# \# \#}<0.001$. IHC, Immunohistochemistry.

2012; www.olfacto-meter.com). In this paradigm, mice were trained to respond to the presence of a rewarded stimulus odorant $\left(\mathrm{S}^{+}\right)$by licking the water delivery tube, and to refrain from responding to the presence of non-rewarded stimulus odorant $\left(\mathrm{S}^{-}\right)$. A response in an $\mathrm{S}^{+}$trial and an absence of response in an $\mathrm{S}^{-}$trial were scored as correct. Accuracy was scored for each block of 20 trials. Mice were subjected to a daily session of eight blocks. Monomolecular odorant compounds and binary odorant mixtures (Sigma-Aldrich), were diluted either in water or in mineral oil (MO), and $10 \mathrm{ml}$ of the resulting solution was used as the odorant source.

Olfactometry was performed in the following order: (1) training and discrimination of isoamyl-acetate (IAA) versus MO (1st and 2nd weeks), (2) odorant ( $N$-butanol) detection threshold (3rd and 4 th weeks), and (3) carvone and carvone mixture discrimination (5th and 6th weeks). Olfactory memory was assessed in one session following a 4 week interval from the end of the 5 th week.

In the odorant detection threshold test, mice were tested for their ability to detect successively descending decimal concentrations of $\mathrm{N}$-butanol $\left(\mathrm{S}^{+}\right)$diluted in water $(\mathrm{S})$, starting with a concentration of $0.01 \%$. The session ceased at the achievement of criterion performance
( $\geq 75 \%$ of correct responses in at least one block). The odorant detection threshold test ceased when the criterion performance was not achieved after three successive sessions with the same odorant dilution. The accuracy criterion ( $\geq 75 \%$ of correct responses in at least 1 block) was used to calculate odorant detection threshold.

In the odorant mixture discrimination tasks, mice were trained to distinguish between two carvone enantiomers diluted in MO: 0.1\% carvone ${ }^{+}$ $\left(\mathrm{S}^{+}\right)$and $0.1 \%$ carvone ${ }^{-}\left(\mathrm{S}^{-}\right)$. Carvones belong to the terpenoid family and are food scents. $\mathrm{S}^{+}$-carvone smells like caraway (Carum carvi) and Anethum graveolens, whereas $\mathrm{S}^{-}$-carvone smells like spearmint. Mammals, including humans, are able to spontaneously discriminate between the two carvone enantiomers (Linster et al., 2002). Mice were then tested in two discrimination tasks including carvone mixtures. In the first task, $\mathrm{S}^{+}$was attributed to a solution of $80 \%$ carvone $^{+}$and $20 \%$ carvone $^{-}$, and $\mathrm{S}^{-}$to a solution of $20 \%$ carvone $^{+}$and $80 \%$ carvone $^{-}$. In the second task, the $\mathrm{S}^{+}$odorant was a solution of $60 \%$ carvone $^{+}$and $40 \%$ carvone $^{-}$, whereas $\mathrm{S}^{-}$was a solution of $40 \%$ carvone $^{+}$and $60 \%$ carvone $^{-}$.

For the olfactory memory task, mice underwent eight blocks of 20 trials every day for 5 consecutive days, to recall distinguishing between 
A

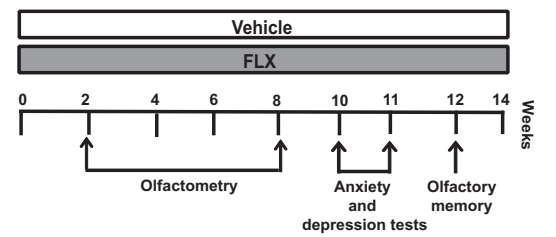

B

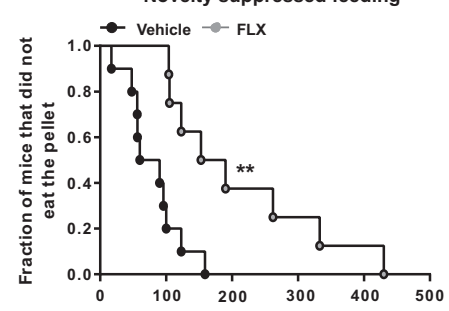

C

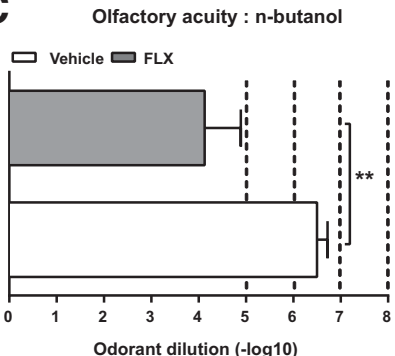

D

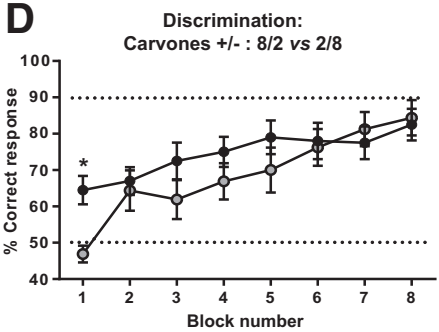

E

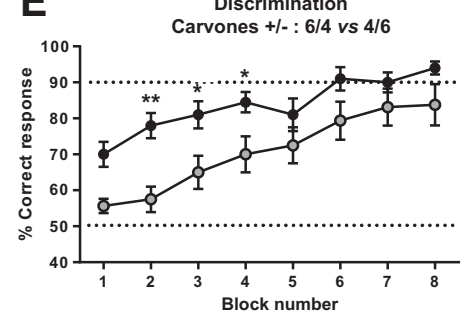

$\mathbf{F}$

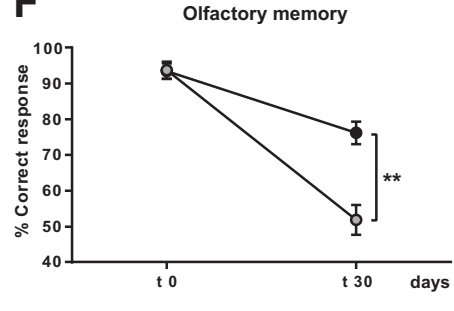

Figure 3. Chronic FLX affects anxiety/depression-like states and impairs olfaction. $\boldsymbol{A}$, Diagram showing experimental groups and study design. $\boldsymbol{B}$, When administered alone, chronic FLX generated some anxiety and depression-related behaviors in otherwise naive mice, mainly an increase in feeding latency in the novelty suppressed feeding paradigm. C, Chronic FLX impaired olfactory acuity since FLX-treated mice required significantly higher concentrations of $\mathrm{N}$-butanol for odorant detection compared with vehicle-treated animals. Mice treated with FLX displayed a poorer learning rate compared with vehicle-treated animals in $\boldsymbol{D}$ the $80 / 20$ versus $20 / 80$ carvone mixture task, albeit reaching the same final score. $E$, In the more difficult discrimination task of $60 / 40$ versus $40 / 60$ carvone mixtures, FLX-treated mice displaying a weaker learning rate as well as a lower final score. $F$, To assess olfactory memory, mice were trained to distinguish between carvone ${ }^{+}$ and carvone ${ }^{-}$during 5 consecutive days. Mice were then retested for the carvone discrimination task at $30 \mathrm{~d}(\mathrm{t} 30)$ following the end of the training session ( $\left.\mathrm{t} 0\right)$. FLX-treated animals exhibited an olfactory memory deficit. Values are plotted as mean \pm SEM ( $n=8-10 /$ group). ${ }^{*} p<0.05,{ }^{* *} p<0.01$.

carvone $^{+}$and carvone ${ }^{-}$. Then, mice were tested on the same task after a rest period of 4 weeks, in one block of 20 trials, but with no reward given for a correct response.

Bromodeoxyuridine injections. Mice were intraperitoneally injected with a DNA synthesis marker, bromodeoxyuridine (BrdU; $100 \mathrm{mg} / \mathrm{kg}$, Sigma-Aldrich). They received one injection $2 \mathrm{~h}$ before perfusion for the analysis of cell proliferation, or four injections, at $2 \mathrm{~h}$ intervals, on a single day, 4 weeks before perfusion, for the analysis of cell survival.

\section{Stereotaxic lentiviral injections}

To study the morphology of adult-born neurons, a replication-deficient lentiviral vector expressing green fluorescent protein (GFP) under control of the cytomegalovirus (CMV) promoter (LV-CMV-GFP with a viral titer of 109 units $/ \mathrm{ml}$ ) was used as previously described (Lazarini et al., 2014). The concentration of lentiviral vector particles used for injection was $13.8 \mathrm{ng}$ of viral antigen per microliter assayed by ELISA $\left(\sim 10^{10}\right.$ transduction units per milliliter). The LV-CMV-GFP vector was prepared at the ENP vector platform (www.paris-neuroscience.fr). Mice were deeply anesthetized by a mixture of ketamine and xylazine (100 $\mathrm{mg} / \mathrm{kg}$ ketamine, $16 \mathrm{mg} / \mathrm{kg}$ xylazine). Small craniotomies were performed above the injection sites using a dentist drill. We performed bilateral virus injections ( $200 \mathrm{nl}$ per site) into the rostral migratory stream (RMS) at $+3.30 \mathrm{~mm}$ anteroposterior and $\pm 0.82 \mathrm{~mm}$ mediolateral from bregma, and $-2.90 \mathrm{~mm}$ dorsoventral from skull surface, to label SVZ-generated neuroblasts that migrate toward the OB. Mice were then transferred to their home cage for recovery.

\section{Immunohistochemistry}

Mice were deeply anesthetized with sodium pentobarbital (i.p., $100 \mathrm{mg} /$ $\mathrm{kg}$, Sanofi) and perfused transcardially with a solution containing $0.9 \%$ $\mathrm{NaCl}$ and heparin $\left(5 \times 10^{3} \mathrm{U} / \mathrm{ml}\right.$, Sanofi-Synthelabo $)$ at $37^{\circ} \mathrm{C}$, followed by $4 \%$ paraformaldehyde in phosphate buffer, $\mathrm{pH} 7.3$. Olfactory epithelia $(\mathrm{OE})$ and brains were removed and postfixed by incubation in the same fixative at $4^{\circ} \mathrm{C}$ overnight. OE were cryoprotected by incubation in $30 \%$ sucrose in PBS for $24 \mathrm{~h}$, and then embedded in Tissue-tek (Sakura). OE $20-\mu \mathrm{m}$-thick coronal sections were obtained using a cryostat (CM3050S, Leica) and were thaw-mounted onto coated glass slides (Superfrost Plus). Forty-micrometer-thick coronal brain sections were obtained using a vibrating microtome (VT1000S, Leica). In the experiments using viral labeling of neuroblasts, $60-\mu \mathrm{m}$-thick coronal sections were prepared. Immunostaining was performed on free-floating sections for brain slices and fixed sections for OE. Nonspecific staining was blocked by $0.2 \%$ Triton, $4 \%$ bovine serum albumin (Sigma-Aldrich) and $2 \%$ goat serum. Slices were first incubated with primary antibodies at $4^{\circ} \mathrm{C}$ overnight, and then with secondary antibodies (biotinylated or AlexaFluor-conjugated secondary antibodies, Jackson ImmunoResearch Laboratories) at room temperature. Primary antibodies used in this study and their working dilutions are listed in Table 1.

For doublecortin (DCX) immunolabeling, brain sections were preincubated in citrate buffer $(10 \mathrm{~mm}, \mathrm{pH} 9.0)$ at $80^{\circ} \mathrm{C}$ for $20 \mathrm{~min}$. For Ki67 immunostaining, OE sections were preincubated in citrate buffer $(10$ $\mathrm{mm}, \mathrm{pH} 6.0$ ) at $80^{\circ} \mathrm{C}$ for $20 \mathrm{~min}$. For BrdU staining, DNA was denatured with $2 \mathrm{~N} \mathrm{HCl}$ at $37^{\circ} \mathrm{C}$ for $30 \mathrm{~min}$. Fluorescent sections were stained with the nuclear dye 4',6'-diamidino-2-phenylindole (DAPI) and then mounted in a solution of 1,4-diazabicyclo [2.2.2] octano (SigmaAldrich). Immunoperoxidase-labeled cells were detected with a peroxidase-conjugated secondary antibody (ABC system, Vector Laboratories), using biotinylated donkey anti-rat, goat anti-rabbit or horse antigoat IgG (1:200, Vector Laboratories) and 3,39-diaminobenzidine $(0.05 \%)$ as a chromogen (Sigma-Aldrich).

For morphologic analysis of adult-born neurons in the OB, the labeling was enhanced with a GFP immunostaining (rabbit anti-GFP primary antibody, 1/1000, and goat anti-rabbit second antibody, 1/500, Invitrogen). DAPI (1/5000) was used as a nuclear stain.

\section{Image acquisition and quantification analysis}

Immunofluorescence was analyzed using a confocal laser-scanning microscope (LSM 710; Zeiss) with Zen Imaging software (Zeiss) and a confocal spinning disk Cell Voyager (CV1000, Yokogawa). For the analysis of immunoperoxidase-labeled cells, reconstructed images of the dentate gyrus (DG), SVZ, and OB were taken using an Olympus BX51 microscope $(20 \times$ objective) and the Compix Imaging software (Hamamatsu Photonics).

Quantification was performed using the Icy open source platform (http://www.icy.bioimageanalysis.org; Olivo-Marin, 2002; de Chaumont et al., 2012). The internal and external borders of the RMS, the glomerular layer (GL), and granule cell layer (GCL) of the $\mathrm{OB}$ were 
A

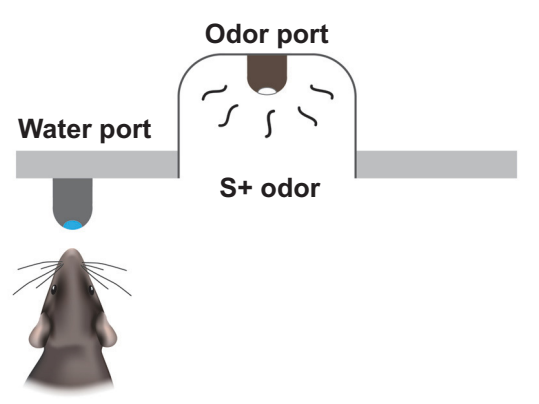

B

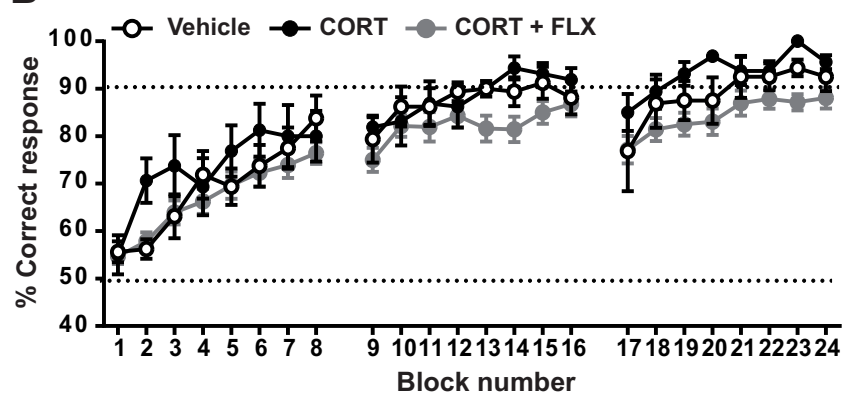

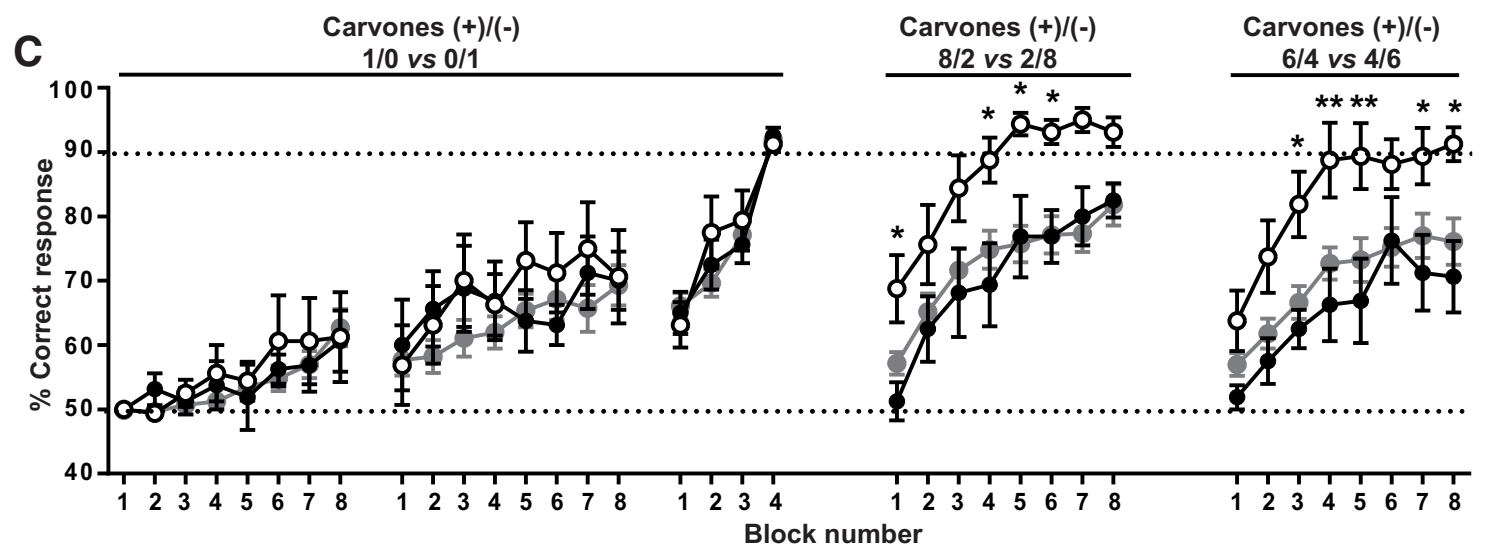

D

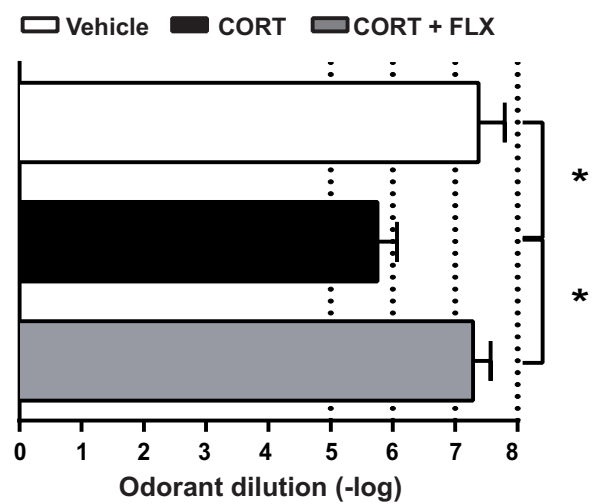

E

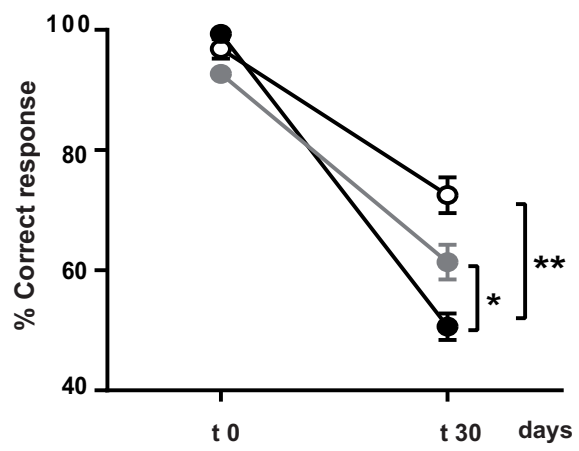

Figure 4. Chronic CORT induces multiple olfactory deficits most of which are restored by FLX. $A$, Go/no-go procedure. In response to a positive odor stimulus $\left(S^{+}\right)$, the trained mouse licks the water port to receive the water reward. $\boldsymbol{B}$, Graph showing the mean percentage of correct responses in each block of the discrimination task between $1 \%$ IAA and $1 \%$ MO . All groups of mice reached criterion performance, which corresponds to $90 \%$ of correct responses in at least one block. A score of $50 \%$ corresponds to the success rate at chance level. C, Graph depicting the mean percentage of correct responses in each block of the discrimination task between two carvone enantiomers and their mixtures. To further evaluate olfactory discrimination, we trained mice to discriminate mixtures of increasing complexity. Mice were first trained to distinguish $1 \%$ carvone ${ }^{+}$and $1 \%$ carvone $^{-}$. Once mice reached this criterion, they were subjected to the more difficult task of mixture discrimination. The mixture ratio of carvone ${ }^{+}$and carvone ${ }^{-}$is indicated on the graph. All groups showed similar performance in the simple discrimination task of carvone ${ }^{+}$versus carvone ${ }^{-}$. However, the ability to discriminate between carvone mixtures was reduced by CORT and not restored by FLX. D, CORT-treated mice required significantly higher concentrations of $N$-butanol for odorant detection compared with vehicle-treated and CORT $+F L X$-treated animals. $E$, To assess olfactory memory, mice were trained during 5 consecutive days to recall distinguishing carvone ${ }^{+}$and carvone ${ }^{-}$. Mice were then retested in the simple carvone discrimination task at $30 \mathrm{~d}(\mathrm{t} 30)$ following the end of the training session (t0). CORT-treated animals exhibited a memory deficit that was attenuated by FLX. Values are plotted as mean \pm SEM $(n=8$ for vehicle and CORT-treated; $n=35$ for CORT $+\mathrm{FLX}) .{ }^{*} p<0.05,{ }^{* *} p<0.01$.

Table 1. Detailed information on the primary antibodies used in this study

\begin{tabular}{llll}
\hline Antibody & Manufacturer & Marker & $\begin{array}{l}\text { Working } \\
\text { dilutions }\end{array}$ \\
\hline BrdU & Abcys, ABC 117-7513 & Proliferating cells & $1: 1000$ \\
DCX & Abcam, ab 18723 & Neuroblasts & $0.5 \mu \mathrm{g} / \mathrm{ml}$ \\
GFP & Molecular Probes & LV-CMV-GFP & $1: 1000$ \\
Ki67 & Abcam, ab16667 & Proliferating cells & $1: 500$ \\
NeuN & Millipore Bioscience Research Reagents, & Neurons & $5 \mu \mathrm{g} / \mathrm{ml}$ \\
OMP & MAB377 & Olfactory sensory neurons & $1: 2000$ \\
& Wako, 544-10001 & & \\
\hline
\end{tabular}

delineated and $\mathrm{DCX}^{+}$and $\mathrm{BrdU}^{+}$cells were counted manually using the plugin "Manual counting" (http://icy.bioimageanalysis.org/plugin/ manual_counting). Regions-of-interest were designed with the ring plugin (http://icy.bioimageanalysis.org/plugin/Ring ROI). Values are expressed as the mean of total $\mathrm{DCX}^{+}$or $\mathrm{BrdU}^{+}$cell count in six $\mathrm{OB}$ slices per animal.

For the quantification of DCX immunoreactivity (IR) in the SVZ and olfactory marker protein (OMP) IR in glomeruli of the OB, pixel density was computed using the plugin "Thresholded pixel density." This plugin computes the number of pixels over a threshold in a given region of interest over the total number of pixels present in the region of interest (http://icy.bioimageanalysis.org/plugin/Thresholded_pixel_density). For OMP 
IR, six glomeruli of the dorsomedial part of OB coronal sections were selected and assessed for each animal. The batch mode of this analysis is available as a protocol [http://icy.bioimageanalysis.org/protocol/Pix- el_density_(batch_mode)]. All the codes and implementation of these programs are available online. For the quantification of $\mathrm{Ki}^{+}{ }^{+}$cells in the $\mathrm{OE}$, serial sections of the olfactory mucosa situated through the anterior end of the $\mathrm{OB}$ were analyzed blindly. Four to six serial sections were chosen per animal ( 5 animals analyzed per group, $6-7$ zones analyzed per OE section).

For hippocampal analysis, values are expressed as the mean total number of $\mathrm{DCX}^{+}$or $\mathrm{BrdU}^{+}$cells counted manually in $8 \mathrm{DG}$ sections per animal. For BrdU and neuronal nuclei $(\mathrm{NeuN})$ double-staining analysis, positive cells were counted in the GCL (6 slices per animal, 4 mice analyzed per group, $80-120 \mathrm{BrdU}^{+}$cells analyzed) and in the DG (8 slices per animal, 4 mice analyzed per group, $20-30 \mathrm{BrdU}^{+}$cells analyzed). Data are expressed as the number of double-labeled cells in the GCL and DG.

For the analysis of dendritic morphology of adult newborn OB neurons, 8 coronal sections of a $180 \mu \mathrm{m}$ distance were selected, taking the accessory $\mathrm{OB}$ as a landmark. Fifteen neurons (located $\geq 40 \mu \mathrm{m}$ in the GCL) were selected for each animal. These neurons were imaged using a confocal microscope (Zeiss LSM 700, $25 \times$ objective, $0.5-1$ numerical zoom). The dendritic tree was manually traced using the Filament tracer module of the Imaris Bitplane software v7.6. Total dendritic length, number of branches and Sholl data were collected. For Sholl analysis, virtual concentric circles were traced at every micrometer starting from the beginning of the dendrite, and the number of intersections between the dendritic tree and each circle were counted as previously described (Pallotto et al., 2012). For the analysis of dendritic morphology of hippocampal adult-born neurons, the maturation index was calculated as previously described (Mendez-David et al., 2014). DCX ${ }^{+}$cells were subcategorized according to their dendritic morphology as $\mathrm{DCX}^{+}$cells without and $\mathrm{DCX}^{+}$cells with tertiary (or higher order) dendrites. The maturation index was defined as the ratio of $\mathrm{DCX}^{+}$cells possessing tertiary dendrites to the total number of $\mathrm{DCX}^{+}$cells.

\section{Statistical analysis}

All data were expressed as mean \pm SEM. Statistical analyses were performed using the Prism software (GraphPad v6), with $p<0.05$ considered statistically significant. Data were analyzed using unpaired two-tailed Student's $t$ test, one-way ANOVA or two-way ANOVA followed by Bonferroni post hoc test or Mantel-Cox log-rank test when appropriate.

\section{Results \\ Chronic CORT treatment induces anxiety- and depression- like states in mice}

Using standard tests for anxiety and depression, we confirmed that chronic treatment with CORT triggers an anxiety/ depression-like phenotype that is responsive to chronic FLX treatment. At 4 weeks of treatment, CORT decreased the time spent in the center of the open field ( $185 \pm 12 \mathrm{~s}$ vs $333 \pm 43 \mathrm{~s}, t=$ $4.53, p<0.001$, unpaired $t$ test; Fig. $1 A$ ), and in the open arms of the elevated plus maze $(39 \pm 3 \mathrm{~s}$ vs $58 \pm 11 \mathrm{~s}, t=2.19, p=0.03$, unpaired $t$ test; Fig. $1 B$ ). In the novelty suppressed feeding test, CORT significantly increased feeding latency ( $359 \pm 23 \mathrm{~s}$ vs $97 \pm$ 12 s, $p<0.001$, Kaplan-Meier survival analysis, Mantel-Cox logrank test; Fig. 1C) without affecting feeding drive (Fig. 1D), whereas in the splash test CORT increased grooming latency $(207 \pm 17 \mathrm{~s}$ vs $55 \pm 12 \mathrm{~s}, p<0.001$, Kaplan-Meier survival analysis, Mantel-Cox log-rank test; Fig. $1 E$ ) and decreased grooming frequency $(2 \pm 0.2$ vs $5 \pm 0.8, t=4.78, p<0.001$, unpaired $t$ test; Fig. $1 F)$.

At 16-17 weeks of CORT treatment (Fig. 2A), feeding latency in the novelty suppressed test remained significantly increased $(470 \pm 52 \mathrm{~s}$ vs $80 \pm 15 \mathrm{~s}, p<0.001$, Kaplan-Meier survival analysis, Mantel-Cox log-rank test; Fig. 2B) without changes in feeding drive (vehicle: $0.29 \pm 0.05$; CORT: $0.25 \pm 0.06$; CORT+FLX: $0.23 \pm 0.07 ; F_{(2,44)}=0.19, p=0.1$, one-way ANOVA, Bonferroni post hoc test; Fig. 2C). Moreover, changes in grooming behavior persisted, namely decreased grooming frequency $\left(3 \pm 0.3\right.$ vs $6 \pm 0.7 ; F_{(2,44)}=7.09, p=0.03$, one-way ANOVA, Bonferroni post hoc test; Fig. $2 D)$ and increased latency to groom $(116 \pm 18 \mathrm{~s}$ vs $29 \pm 7 \mathrm{~s}, p<0.001$; Kaplan-Meier survival analysis, Mantel-Cox log-rank test; Fig. 2E). CORT also increased immobility in the forced swim test $(119 \pm 18 \mathrm{~s}$ vs $230 \pm$ $37 \mathrm{~s} ; F_{(2,44)}=4.13, p=0.02$, one-way ANOVA, Bonferroni post hoc test; Fig. $2 F$ ) and in the tail suspension test, albeit in a nonsignificant manner (Fig. 2G). Chronic FLX exerted antidepressant effects that were mainly prominent in the novelty suppressed feeding and splash tests. More specifically, FLX restored feeding latency (176 $\pm 34 \mathrm{~s}, p<0.001$ vs CORT, one-way ANOVA, Bonferroni post hoc test), as well as grooming frequency $(6 \pm 0.5, p=0.002$ vs CORT, one-way ANOVA, Bonferroni post hoc test) and latency to groom ( $27 \pm 4 \mathrm{~s}, p<0.001$ vs CORT, Kaplan-Meier survival analysis, Mantel-Cox log-rank test; Fig. $2 B, D, E$ ). When administered alone for $10-11$ weeks (Fig. $3 A$ ), chronic FLX generated some anxiety-related behaviors in otherwise naive mice, reflected mainly by an increase in feeding latency in the novelty suppressed feeding test (vehicle: $81 \pm 13 \mathrm{~s}$; FLX: $212 \pm 42 \mathrm{~s}, t=3.3, p=0.005$, unpaired $t$ test; Fig. $3 B$ ). Together, these data indicate that chronic CORT exposure induces an anxiety/depression-like state in mice that is reversed by prolonged coadministration of FLX.

\section{Olfactory discrimination is impaired by chronic CORT and not restored by FLX}

To test whether the observed anxiety/depression-like state was accompanied by olfactory deficits, we first evaluated olfactory discrimination by using an automated operant conditioning procedure (a go/no-go test; Fig. 4A). Mice were trained to recognize IAA as the stimulus rewarded with water $\left(\mathrm{S}^{+}\right)$and $\mathrm{MO}$ as the non-rewarded stimulus $\left(\mathrm{S}^{-}\right.$; Fig. $4 B$ ). In the next step, mice were trained to distinguish between two carvone enantiomers diluted in MO; carvone ${ }^{+}$ $\left(\mathrm{S}^{+}\right)$and carvone ${ }^{-}\left(\mathrm{S}^{-}\right)$; and we then assessed fine olfactory discrimination using binary mixtures of the same carvone enantiomers (Fig. 4C). We observed that CORT and CORT+FLX did not affect acquisition and success rates in the simple discrimination task of IAA versus $\mathrm{MO}$ and in the carvone enantiomer task (Fig. 4B,C). However, in the "difficult" mixture discrimination task, the performance of CORT-treated animals was significantly poorer compared with controls and was not restored by FLX (two-way repeated mesures ANOVA, $8 / 2$ mixture ratio task: treatment factor $F_{(2,44)}=5.04, p=$ $0.01 ; 6 / 4$ mixture ratio task: treatment factor $F_{(2.44)} \stackrel{=}{=} .43, p=0.01$; Fig. 4C).

\section{Olfactory acuity is impaired by chronic CORT and restored by FLX}

Mice were trained to detect successively descending decimal concentrations of $\mathrm{N}$-butanol $\left(\mathrm{S}^{+}\right)$diluted in water $\left(\mathrm{S}^{-}\right)$, starting with a concentration of $0.01 \%$. CORT decreased the detection threshold by two orders of magnitude (Fig. $4 D$; mean odorant dilution $(-\log )$; vehicle: $8.2 \pm 0.4$; CORT: $6.5 \pm 0.3 ; F_{(2.44)}=$ $4.23, p=0.02$, one-way ANOVA, Bonferroni post hoc test). Cotreatment with FLX attenuated the CORT-induced deficit (Fig. $4 D$; mean odorant dilution $(-\log ) ; 7.7 \pm 0.3, p=0.03$, one-way ANOVA, Bonferroni post hoc test). 


\section{Olfactory memory is affected by chronic CORT and improved by FLX}

We assessed long-term olfactory memory by using the same pair of carvone isoforms (Fig. 4E). Thirty days following task consolidation, the ability of mice to recognize carvone ${ }^{+}$and carvone ${ }^{-}$ enantiomers was re-evaluated, using one block of 20 trials that was devoid of water reward. Four weeks following task consolidation, the performance of CORT-treated mice was near chance level, revealing that chronic CORT impaired olfactory memory [mean correct response; vehicle: $72.5 \%$; CORT: $50.6 \%$; $p=$ 0.002 , two-way ANOVA; $F_{(2.44)}$ (treatment $\times$ memory $)=7.22$ ]. Treatment with FLX was able to improve performance (mean correct response; $61.3 \%, p<0.05$ compared with CORT).

\section{Olfaction is impaired by chronic FLX}

Although FLX restored most CORT-induced olfactory deficits, it significantly impaired olfaction when administered alone chronically (Fig. 3C-F). FLX induced a substantial decrease in olfactory acuity [mean odorant dilution $(-\log )$; vehicle: $6.5 \pm 0.2$; CORT: $4.1 \pm 0.8 ; t=3.28, \mathrm{df}=16, p=0.003$, unpaired $t$ test; Fig. $3 C]$. Fine discrimination was also affected (Fig. $3 D, E$ ), and this effect was most prominent in the $6 / 4$ versus $4 / 6$ carvone mixture task (two-way ANOVA; $F_{(1,16)}$ treatment $=8.59, p=0.01$; Fig. $3 E$ ). Long-term olfactory memory was also significantly impaired by FLX (two-way ANOVA; $F_{(1,32)}$ interaction $=16.45, p=0.003$; Fig. $3 F)$.

\section{Adult neurogenesis is impaired by chronic CORT and restored by chronic FLX}

We then investigated whether adult neurogenesis was affected by CORT. We measured the production of new neurons in the SVZ and DG and their survival rate in the $\mathrm{OB}$ and DG. Quantitative analysis of the population of adult-born neurons at 20 weeks of CORT and 14 weeks of CORT+FLX treatment showed a significant twofold decrease in the number of $\mathrm{DCX}^{+}$neurons in the DG that was reversed by FLX (mean cell number; vehicle: $4782 \pm$ 115; CORT: $1879 \pm 286$; CORT+FLX: $7439 \pm 910$ cells; $F_{(2.18)}=$ 7.06; CORT vs vehicle: $p=0.04$; CORT vs CORT+FLX: $p=$ 0.002; one-way ANOVA, Bonferroni post hoc test; Fig. $5 A, D$ ). On the other hand, neither CORT nor CORT + FLX altered the number of $\mathrm{DCX}^{+}$in the SVZ (Fig. $5 B, F$ ). These results indicate that CORT affects differentially the two stem cell niches of the adult brain, reducing neural production in the DG but sparing SVZ neurogenesis. Yet, we found that the number of $\mathrm{DCX}^{+}$neurons in the core of the OB, the RMSob, was decreased in the CORT group, and this effect was reversed by FLX (vehicle: 15,834 \pm 1049; CORT: 10,316 \pm 310; CORT+FLX: 15,590 \pm 1332 cells; $F_{(2.18)}=5.78$; CORT vs vehicle: $p=0.04$; CORT vs CORT+FLX: $p=0.03$, one-way ANOVA, Bonferroni post hoc test; Fig. $5 C, H)$. Moreover, the number of $\mathrm{DCX}^{+}$cells was notably reduced by CORT and restored by FLX in the GCL (vehicle: 29,292 \pm 1159 ; CORT: $15,876 \pm 355$; CORT+FLX: 29,545 \pm 4557 cells; $F_{(2.18)}=$ 4.02; CORT vs vehicle: $p=0.05$; CORT vs CORT+FLX: $p=0.05$, one-way ANOVA, Bonferroni post hoc test; Fig. $5 C, G)$ and in the GL (vehicle: $2491 \pm 64$; CORT: $1387 \pm$ 158; CORT+FLX: $2476 \pm 282 ; F_{(2.18)}=5.72$; CORT vs vehicle: $p=0.04$; CORT vs CORT+FLX: $p=0.03$, one-way ANOVA, Bonferroni post hoc test; Fig. 5I). Therefore, it is likely that CORT affects olfactory adult neurogenesis by altering neuronal survival in the $\mathrm{OB}$, while leaving intact cell production in the SVZ.

To address this question more directly, we examined both cell proliferation and survival in the DG and $\mathrm{OB}$, by measuring $\mathrm{BrdU}^{+}$cells $2 \mathrm{~h}$ and 1 month following BrdU injection, respec- tively (Fig. 6A). BrdU ${ }^{+}$cell counting confirmed that CORT significantly decreases cell proliferation in the DG, whereas FLX completely restores it (mean $\mathrm{BrdU}^{+}$cell number; vehicle: $723 \pm$ 60; CORT: $414 \pm 58$; CORT +FLX: $985 \pm 126$ cells; $F_{(2.18)}=5.76$; CORT vs vehicle: $p=0.05$; CORT vs CORT +FLX: $p=0.02$, one-way ANOVA, Bonferroni post hoc test; Fig. $6 B, F$ ). However, neither CORT nor CORT + FLX altered the number of $\mathrm{BrdU}^{+}$in the SVZ (Fig. 6G).

Moreover, CORT induced a twofold decrease in the survival of newborn cells in the $\mathrm{DG}\left(\mathrm{BrdU}^{+}\right.$cell number; vehicle: $361 \pm$ 30; CORT: $207 \pm 29 ; F_{(2.18)}=0.58, p=0.02$, one-way ANOVA, Bonferroni post hoc test; Fig. $6 H$ ). FLX reversed this effect albeit in a nonsignificant manner. Likewise, cell survival was reduced by CORT in the $\mathrm{OB}$, both in the $\mathrm{GL}\left(\mathrm{BrdU}^{+}\right.$cell number; vehicle: $997 \pm 123$ cells; CORT: $523 \pm 60$ cells; $F_{(2.18)}=1.17, p=0.05$, one-way ANOVA, Bonferroni post hoc test; Fig. 6I) and the GCL $\left(\mathrm{BrdU}^{+}\right.$cell number; vehicle: $9082 \pm 1212$ cells; CORT: $4978 \pm$ 475 cells; $F_{(2.18)}=4.91, p=0.02$, one-way ANOVA, Bonferroni post hoc test; Fig. $6 \mathrm{~J}$ ). FLX reversed this effect mainly in the GL $\left(\mathrm{BrdU}^{+}\right.$cell number, $985 \pm 101$ cells, $p=0.03$ vs CORT; oneway ANOVA, Bonferroni post hoc test). Finally, BrdU and NeuN colabeling showed that the vast majority of $\mathrm{BrdU}^{+}$cells were also colabeled with the neuronal biomarker NeuN in all treatment groups tested (Fig. 6E,K). In the GCL of OB, analysis of 80-120 cells per animal ( $n=4$ mice) in the vehicle group showed that $\sim 90 \%$ BrdU + cells coexpressed NeuN and thus had a neuronal phenotype. We found that the percentage of $\mathrm{BrdU}^{+}$cells labeled with NeuN was not modified by CORT and CORT + FLX treatment (Fig. 6K; vehicle: $92.5 \pm 1.5 \%$; CORT: $92.8 \pm 0.9 \%$; CORT+FLX: $91.1 \pm 0.6 \%)$. Moreover, we found no group difference in BrdU/NeuN double staining in the DG (20-30 cells per animal; vehicle: $91.1 \pm 0.5 \%$; CORT: $87.7 \pm 3.1 \%$; CORT+FLX: $91.8 \pm 0.7 \%)$. Together, these data indicate that chronic CORT exposure affects both adult hippocampal and olfactory neurogenesis and that chronic FLX can stimulate the recruitment and/or survival of newborn neurons within the DG and OB.

\section{Chronic CORT treatment does not alter adult neurogenesis in the olfactory epithelium}

Given the prominent effects of CORT on olfactory function, we checked whether the olfactory sensory organ could be impaired. Olfactory sensory neurons located in the OE project via the olfactory nerve to glomeruli in the GL of the $\mathrm{OB}$, where they synapse on the dendrites of output mitral/tufted cells. Olfactory sensory neurons are continually replaced throughout life by the progeny of dividing basal cells located in the OE. We quantified the level of cell proliferation in the OE by counting the number of $\mathrm{Ki}^{+}$ cells in this zone. Anti-Ki67 immunostaining is mainly localized in the basal area of the normal OE (Fig. $7 A, B$ ). We found no difference in the distribution and the number of $\mathrm{Ki}^{+}{ }^{+}$cells between vehicle and CORT-treated mice following 4 weeks of treatment [mean number of $\mathrm{Ki}^{+} 7^{+}$cells $\left(10^{4}\right)$ per $\mathrm{mm}^{3}$; vehicle: $8.09 \pm 0.23$; CORT: $8.55 \pm 0.65$, unpaired $t$ test; Fig. $7 C$ ]. Staining for OMP, a cell marker specific of mature olfactory sensory neurons, was quantified in the GL (Fig. 7E). We found no difference in the distribution and intensity of OMP between vehicle, CORT and CORT+FLX-treated mice (mean IR within glomeruli; vehicle: $0.1498 \pm 0.02$; CORT: $0.1803 \pm 0.01$; CORT + FLX: $0.1503 \pm 0.01$; one-way ANOVA, Bonferroni post hoc test; Fig. $7 F)$. These results indicate that cell proliferation in the $\mathrm{OE}$ and the degree of sensory innervation in the $\mathrm{OB}$ are both insensitive to chronic CORT treatment. 
A

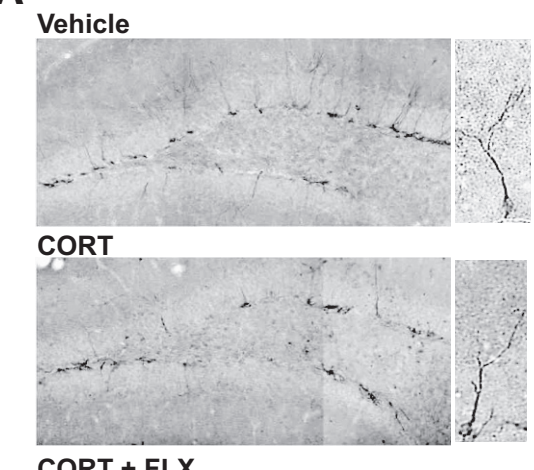

CORT + FLX

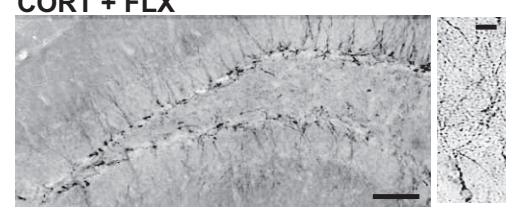

D

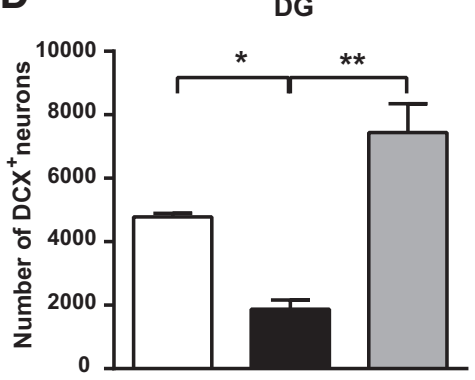

G

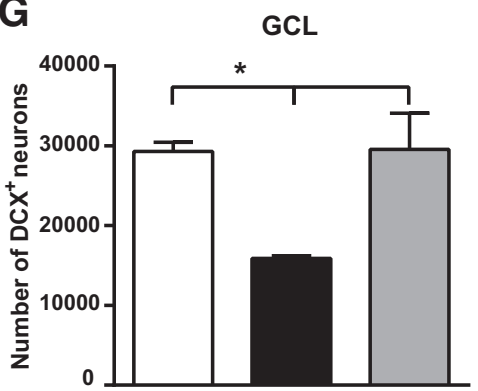

B

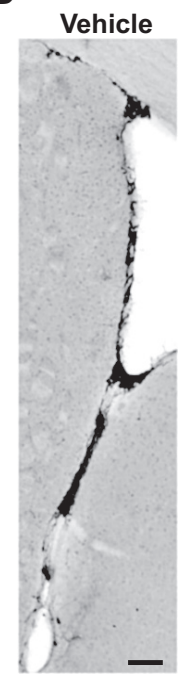

E

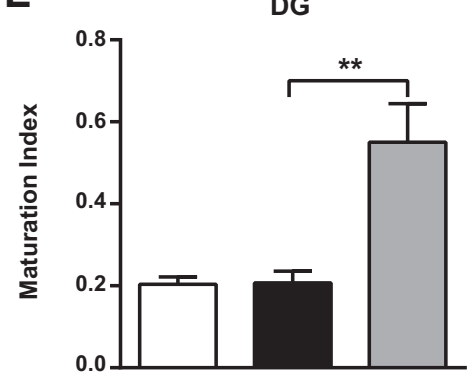

H

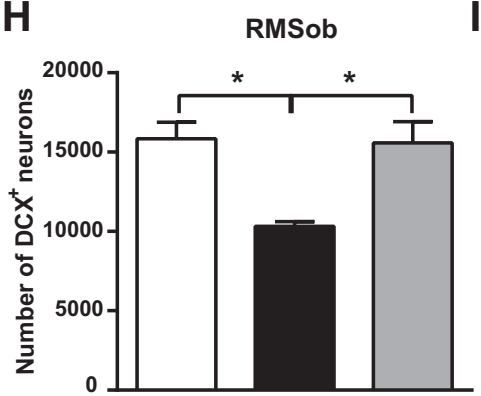

C
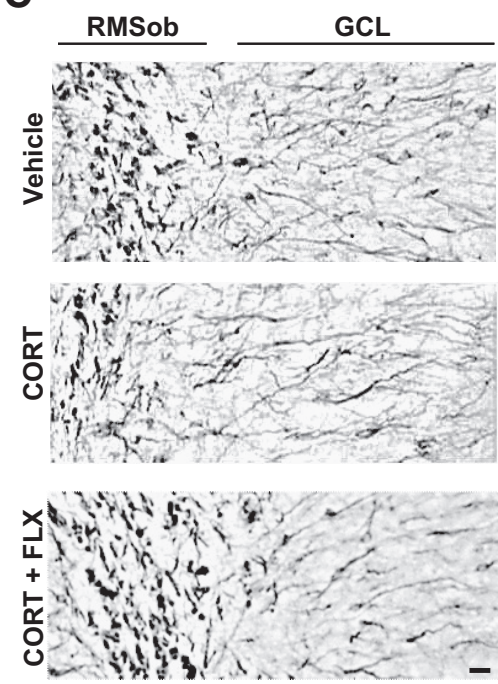

F

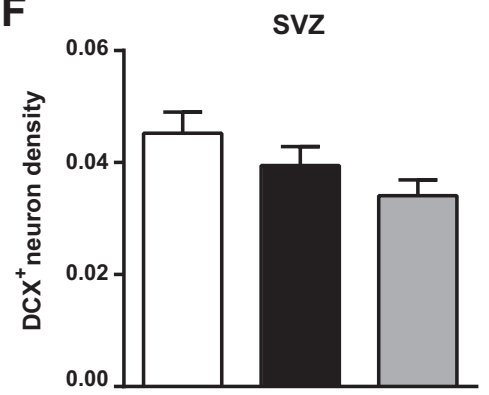

I

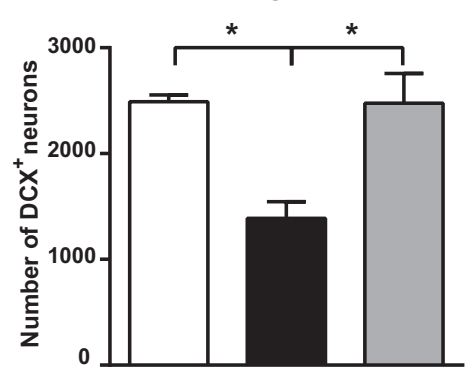

Figure 5. Neuronal turnover in the $O B$ and DG is decreased by chronic CORT and restored by FLX. $A$, Representative images showing DCX ${ }^{+}$cells in the DG of the hippocampus. Scale bars: 100 and $10 \mu \mathrm{m} . \boldsymbol{B}$, Representative images of DCX ${ }^{+}$cells in the SVZ (scale bar, $100 \mu \mathrm{m}$ ) and $(C)$ the RMSob and the GCL of the OB (scale bar, $15 \mu \mathrm{m}$ ). Quantitative evaluation of the effects of CORT and CORT + FLX on the number of DCX+ cells in the $(\boldsymbol{D}) \mathrm{DG},(\boldsymbol{F}) \mathrm{SVZ},(\boldsymbol{G}) \mathrm{GCL}$ of the $\mathrm{OB},(\boldsymbol{H})$ RMSob, and $(\boldsymbol{I}) \mathrm{GL}$ layer of the OB. $\boldsymbol{E}$, Maturation index of newborn DG granule cells following CORT and CORT + FLX treatments. The maturation index is calculated as the ratio of $\mathrm{DCX}{ }^{+}$cells with tertiary (or higher order) dendrites to the total number of $D C X^{+}$cells. Values are plotted as mean \pm $\operatorname{SEM}(n=5$ for vehicle and CORT-treated; $n=10$ for CORT $+\mathrm{FLX}) .{ }^{*} p<0.05,{ }^{* *} p<0.01$. RMSob, Rostral migratory stream of the olfactory bulb.

\section{Impact of CORT and FLX on the morphology of newborn interneurons}

Dendritic complexity of adult-born DG neurons increases during the first month following neuronal birth, a stage during which cells express the DCX marker. To further examine the effects of CORT on dendritic morphology of newborn neurons, we characterized $\mathrm{DCX}^{+}$cells in the DG according to the number of dendrites, and calculated the maturation index as the ratio of $\mathrm{DCX}^{+}$cells with tertiary (or higher order) dendrites to the total number of $\mathrm{DCX}^{+}$cells. Although we found no effect of chronic CORT on the maturation index of adult-born DG neuroblasts, we observed a significant threefold increase induced by CORT+FLX (mean maturation index; vehicle: $0.20 \pm 0.02$; CORT: $0.21 \pm 0.03$; CORT+FLX: $0.55 \pm 0.09 ; F_{(2.12)}=$
0.89; CORT vs CORT + FLX: $p=0.007$, one-way ANOVA, Bonferonni post hoc test; Fig. 5E).

DCX is also expressed by adult-born OB interneurons for $<10$ $\mathrm{d}$ after birth but cannot be used for the assessment of dendrite complexity due to the large staining in the OB. Thus, to assess the impact of chronic CORT and FLX on the maturation of newborn OB interneurons, a lentiviral vector expressing the GFP was injected into the RMS following 8 weeks of CORT and 4 weeks of CORT+FLX or FLX alone (Fig. 8A,C). Three weeks after injection, we analyzed the apical dendritic tree of $\mathrm{GFP}^{+}$adult-born granule cells in the OB and found that chronic CORT did not alter the number of dendrites (Fig. $8 D$ ) nor total dendritic length (Fig. 8E). However, CORT+FLX and FLX alone increased both the number of dendrites (vehicle: $4.93 \pm 0.29$; CORT: $4.92 \pm$ 
A
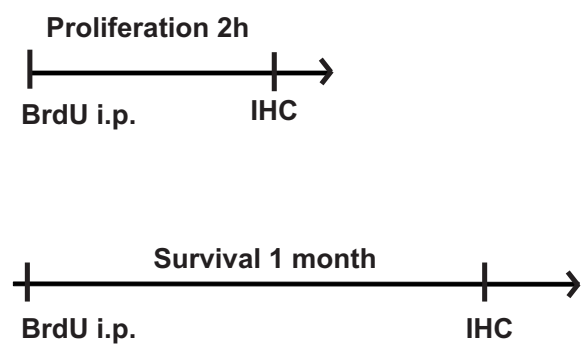

C

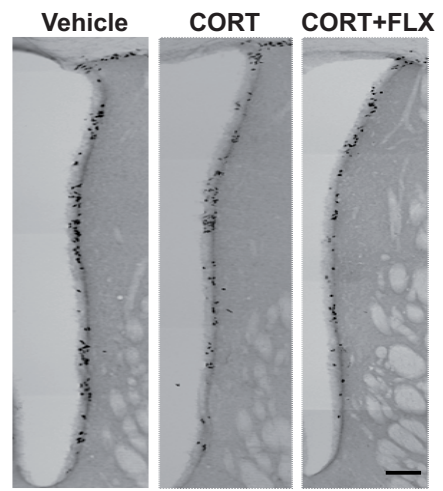

F

Cell proliferation in the DG

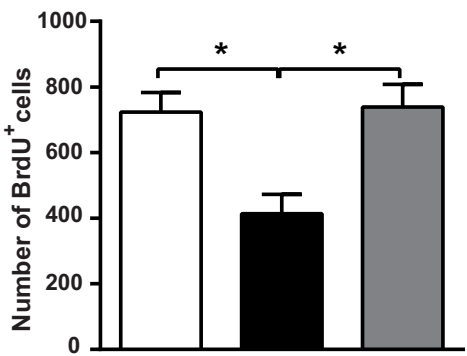

I

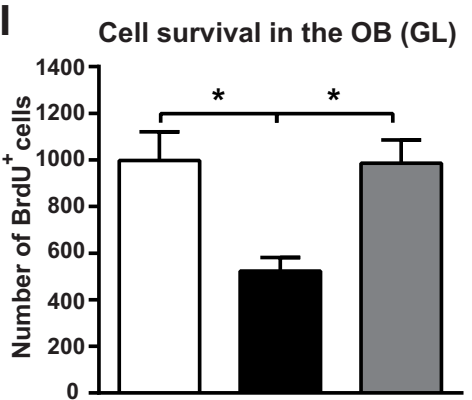

B
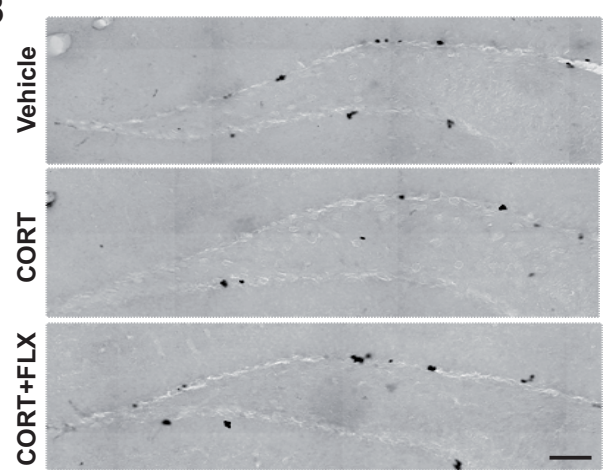

D $\quad$ GCL (OB)
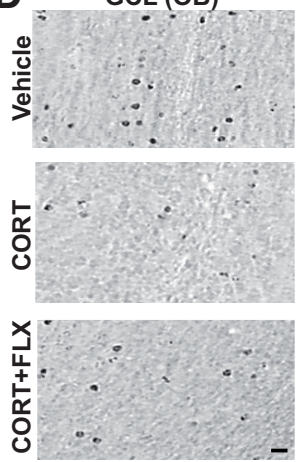

E BrdU

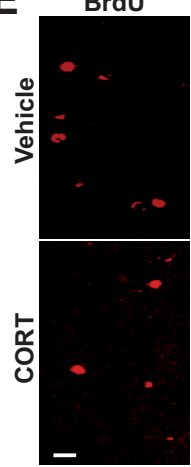

NeuN

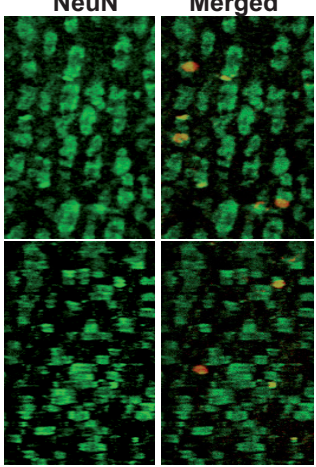

Cell proliferation in the SVZ $\mathrm{H}$
Cell survival in the DG

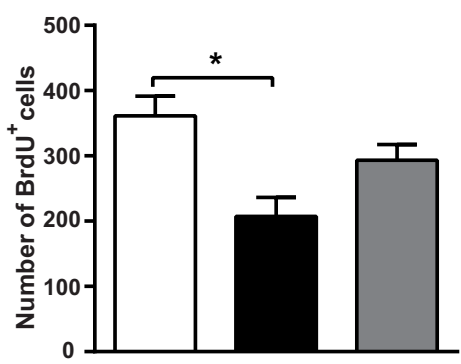

$J$ Cell survival in the $\mathrm{OB}(\mathrm{GCL})$
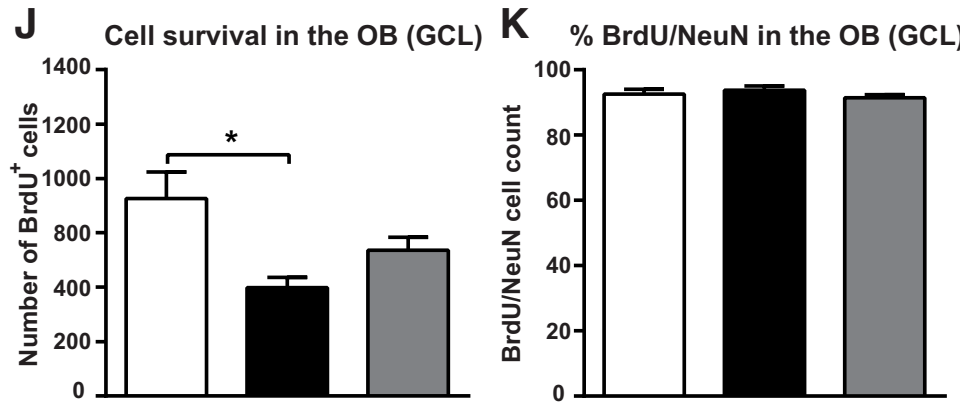

Figure 6. Adult-born neuron proliferation and survival is decreased by chronic CORT and restored by FLX.A, Diagram depicting BrdU injection protocols for the evaluation of cell proliferation and survival. $\boldsymbol{B}, \boldsymbol{C}$, Representative coronal images of BrdU ${ }^{+}$cells in (B) the DG of the hippocampus (scale bar, $100 \mu \mathrm{m}$ ), and (C) the SVZ (scale bar, $100 \mu \mathrm{m}$ ) $2 \mathrm{~h}$ following BrdU administration. $\boldsymbol{D}$, Representative images showing BrdU ${ }^{+}$cells in the $\mathrm{GCL}$ of the $\mathrm{OB}$ of vehicle, CORT and CORT + FLX-treated mice, one month after BrdU administration. Scale bar, $15 \mu \mathrm{m}$. E, BrdU (red) and NeuN (green) colabeled cells in the GCL of vehicle and CORT-treated mice, one month after BrdU administration. Scale bar, $15 \mu \mathrm{m}$. $\boldsymbol{F}, \mathbf{G}$, Graphs depicting the effects of CORT and CORT + FLX on cell proliferation in $(\boldsymbol{F})$ the DG and $(\boldsymbol{H})$ the SVZ ( $n=5-10 /$ group). $\boldsymbol{H}-\boldsymbol{J}$, Graphs showing the effect of CORT and CORT + FLX on cell survival in $(\boldsymbol{H})$ the DG, $(\boldsymbol{I})$ the $G L$ of the OB a,nd $(\boldsymbol{J})$ the $G C L$ of the OB ( $n=5-10 /$ group). $\boldsymbol{K}$, Graph showing the percentage of BrdU ${ }^{+}$cells labeled with NeuN in the GCL of vehicle, CORT and CORT + FLX-treated mice 1 month following BrdU administration ( $n=4-5$ animals per group, $80-120$ cells analyzed per group). Values are plotted as mean \pm SEM. ${ }^{*} p<0.05$.

0.25; CORT +FLX: $5.66 \pm 0.36$; FLX: $5.84 \pm 0.34 ; F_{(3.16)}=0.46$; vehicle vs FLX: $p=0.05$; one-way ANOVA, Bonferroni post hoc test) and dendritic length (vehicle: $559.8 \pm 28.2 \mu \mathrm{m}$; CORT: $586.4 \pm 24.3 ;$ CORT+FLX: $714.7 \pm 24.3 ;$ FLX: $659.8 \pm 25.7$; $F_{(3.16)}=6.76$; vehicle vs CORT+FLX: $p=0.001$; vehicle vs FLX: $p=0.05$; CORT vs CORT+FLX: $p=0.01$; one-way ANOVA,
Bonferroni post hoc test; Fig. $8 D, E)$. Sholl analysis revealed that the most significant changes in the number of branches were obtained by CORT +FLX, suggesting a cumulative effect of the two treatments (two-way ANOVA; $F_{(3,155)}=7.36, p<0.001$; Fig. $8 F$ ). Finally, we observed no effect of CORT and FLX on spine density and length (data not shown). Together, these results sug- 
A

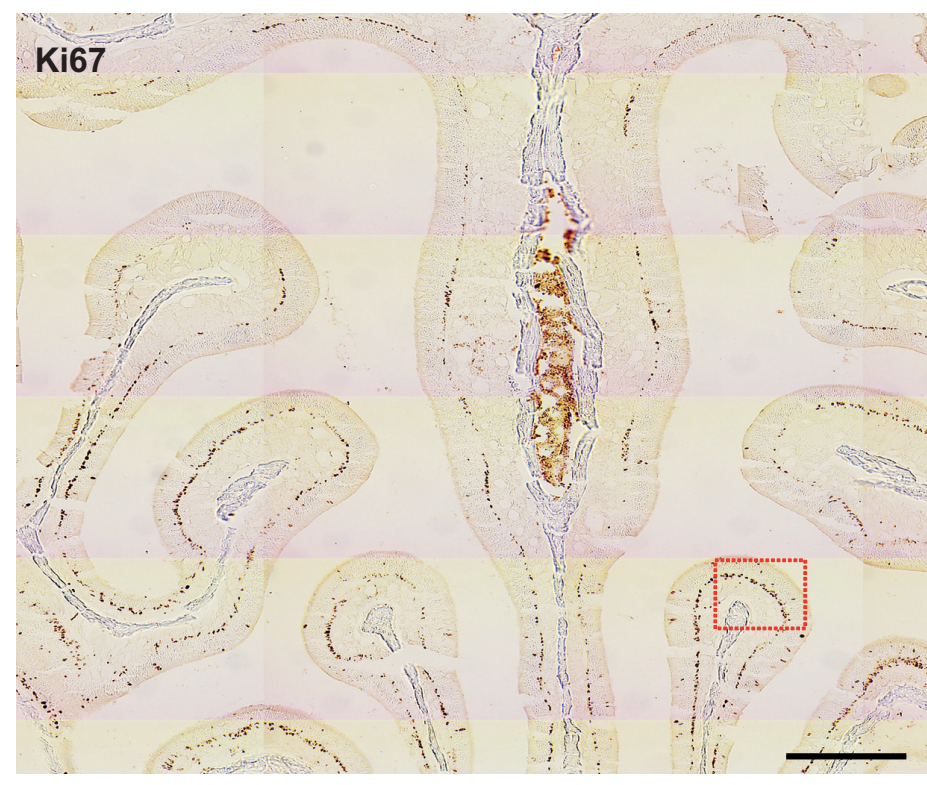

D

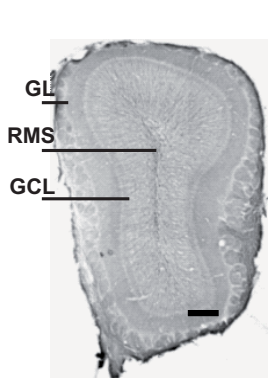

E

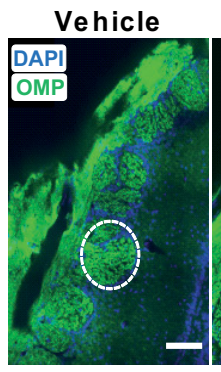

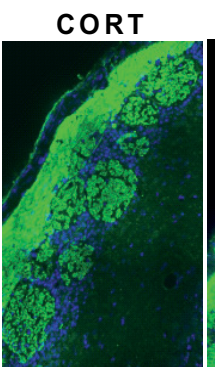

B

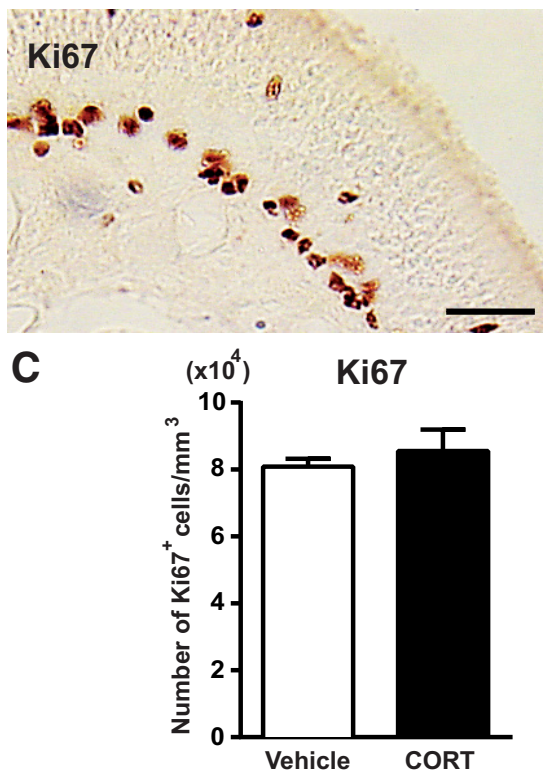

$\mathbf{F}$
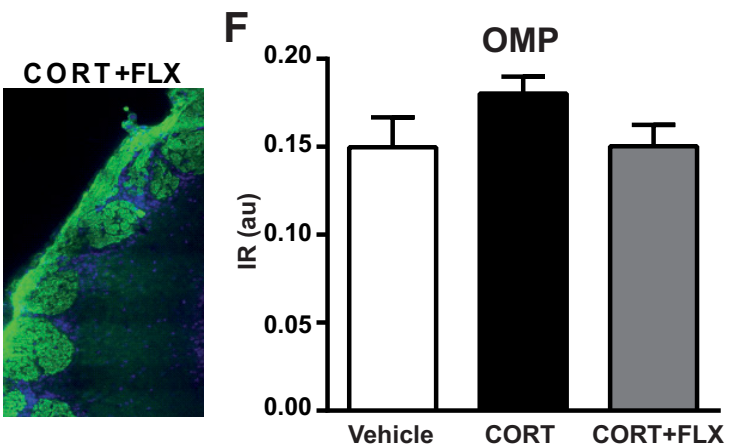

Figure 7. Chronic CORT treatment does not affect sensory afferentation to the $0 B$. $\boldsymbol{A}, \boldsymbol{B}$, Immunostaining with the Ki67 antibody in a normal 8-week-old mouse olfactory mucosa. $\boldsymbol{B}$, A magnification of the region delineated by a red rectangle in $\boldsymbol{A}$. Scale bars: $\boldsymbol{A}, 500 \mu \mathrm{m} ; \boldsymbol{B}, 50 \mu \mathrm{m}$. $\boldsymbol{C}$, Four weeks of $\mathrm{CORT}$ administration did not change the number of Ki67 ${ }^{+}$cells in the $0 \mathrm{E}(n=5$ mice per group). D, OB coronal section of a vehicle-treated mouse, Scale bar, $100 \mu \mathrm{m}$. $\boldsymbol{E}$, Representative images of OMP (green) and DAPI (blue) staining in the GL following 20 weeks of CORT and 14 weeks of CORT + FLX treatment. Scale bar, $50 \mu \mathrm{m}$. The dotted line delineates one glomerulus. $F$, CORT and CORT + FLX had no effect on OMP IR in the OB (values are plotted as mean \pm SEM, $n=$ 4 mice per group; 6 glomeruli in the dorsomedial GL analyzed per animal). au, Arbitrary units.

gest that FLX, but not CORT, facilitates the maturation of newborn neurons in both the adult hippocampus and $\mathrm{OB}$ circuits.

\section{Discussion}

This study reports that a mouse model that mimics states of HPA axis hyperactivity, a common trait of MDD, is associated with impaired adult neurogenesis and pronounced olfactory deficits, including acuity, discrimination, and memory. We found that prolonged antidepressant treatment with fluoxetine not only restored adult neurogenesis and mood, but also improved most olfactory functions. Our data identify a key role for the HPA axis in olfactory impairments associated with anxiety/depressive states.

\section{Deleterious impact of chronic CORT on adult neurogenesis and olfaction}

Our present findings demonstrate that the CORT model not only evokes a persistent fear-related response inhibition, reminiscent of anxiety/depression-like states, but also induces olfactory dysfunction. Importantly, similar olfactory deficits affecting odorant detection and discrimination have been reported in MDDs (Pause et al., 2001; Negoias et al., 2010). In turn, perturbations of the olfactory circuit and function can induce anxiety and depression-like behaviors in rodents (Glinka et al., 2012; Lazarini et al., 2012). Thus, we cannot rule out the possibility that CORTinduced olfactory deficits could be in part responsible for the mood states observed in this model. For instance, olfactory bulbectomy constitutes a model of depression and is used as a predictor of antidepressant drug response in rodents (Wren et al., 1977). Although the precise mechanisms remain elusive, it can be speculated that such sensory deficits likely affect the function of neural circuits involved in the control of mood and emotions.

Interestingly, we also found that chronic CORT significantly reduces neurogenesis in the $\mathrm{OB}$. The number of new $\mathrm{OB}$ interneurons was decreased twofold following CORT treatment in all OB layers. This decrease could partly explain the CORTinduced olfactory deficits, because adult-born OB interneurons take part in odorant detection, discrimination, olfactory learning, and memory (Breton-Provencher et al., 2009; Moreno et al., 2009; Mouret et al., 2009; Belnoue et al., 2011; Lazarini et al., 2011; Alonso et al., 2012; Sakamoto et al., 2014). However, it is important to note that in humans, MDD-related hyposmia is unlikely to be due to reduced neurogenesis in the OB, as cells 
A

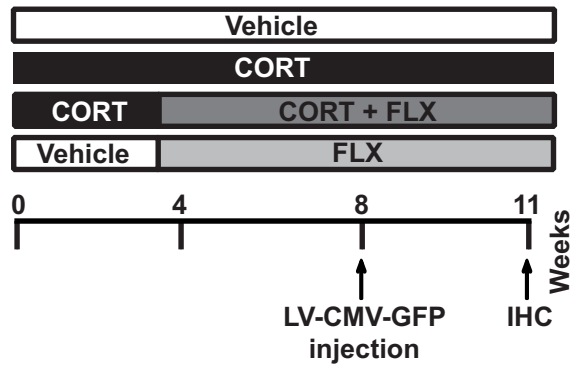

C

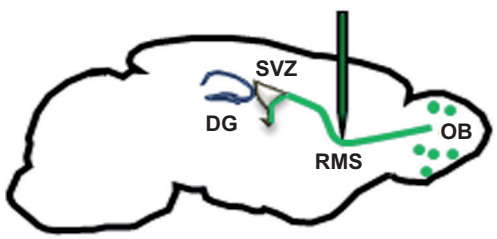

D
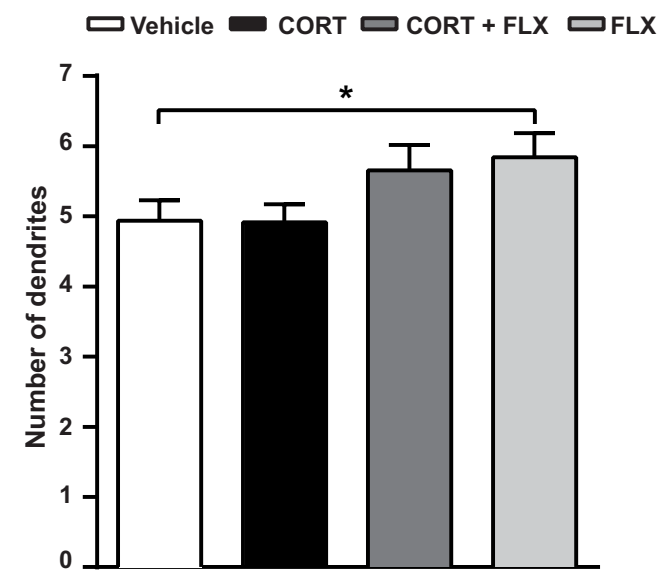

B
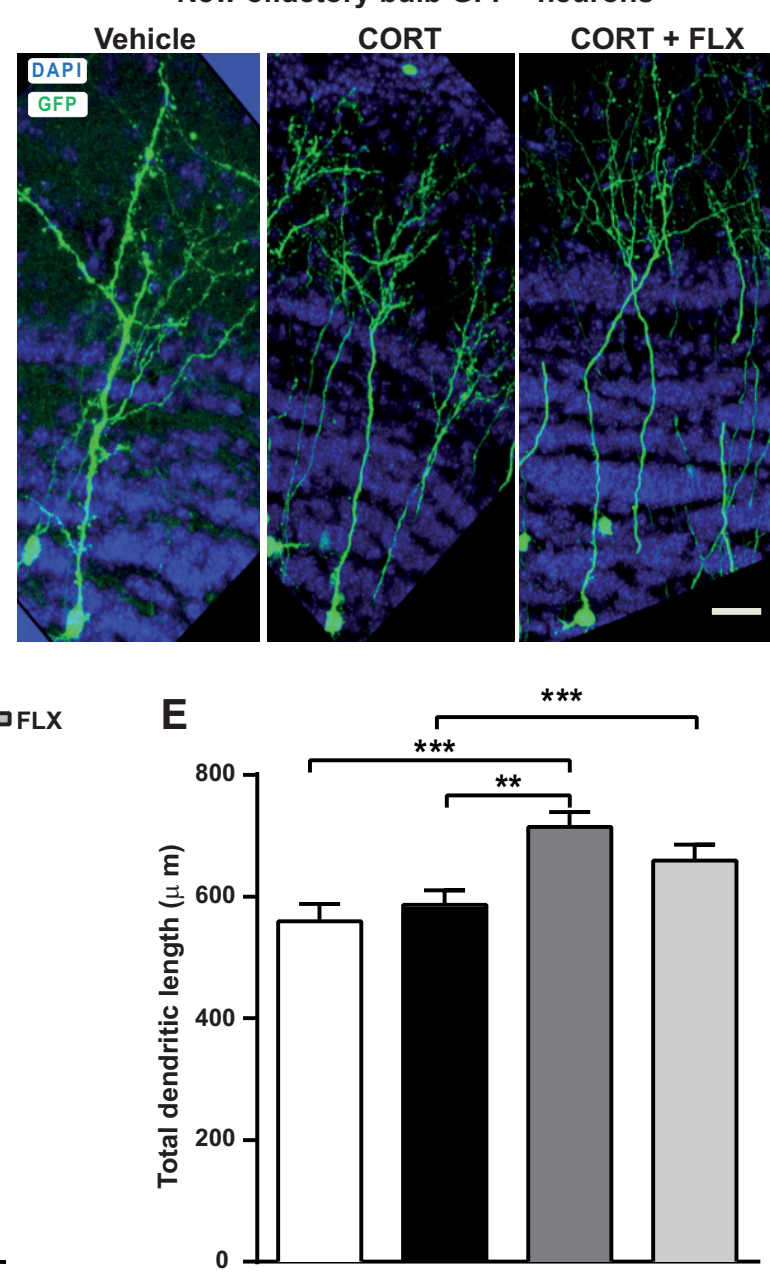

$\mathbf{F}$

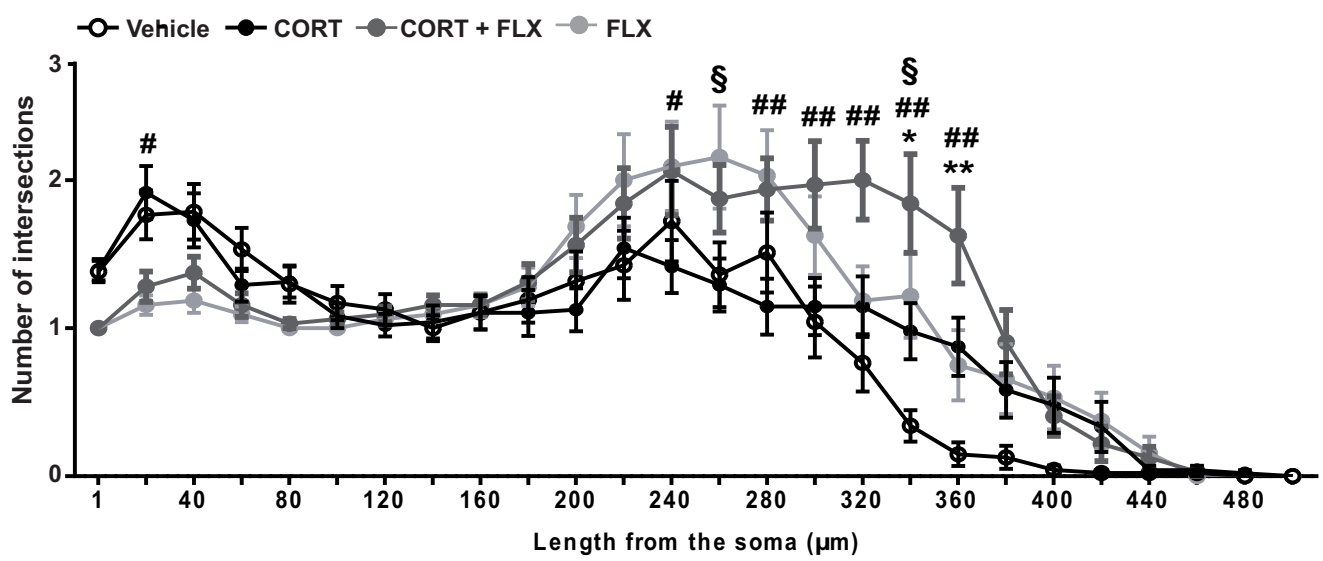

Figure 8. Dendritic length and complexity are increased by chronic CORT and FLX treatments. $A$, Experimental timeline of viral injections and analysis. $B$, Confocal images of vehicle, CORT, and CORT + FLX-treated OB slices showing GFP ${ }^{+}$neurons and DAPI staining. Scale bar, $20 \mu \mathrm{m}$. C, Lentiviral vectors encoding GFP under the control of the CMV promotor were injected in the RMS. D, FLX treatment alone increased the number of dendrites in newborn OB neurons. $E, C O R T+F L X$ and $F L X$ treatments significantly increased dendritic length. $F$, Graph depicting the effect of chronic CORT, CORT + FLX and FLX treatments on the number of dendritic branches, according to Sholl analysis. CORT +FLX treatment increased the number of branches. Values are plotted as mean \pm SEM ( $n=4$ /group). ${ }^{*} p<0.05,{ }^{* *} p<0.01,{ }^{* * *} p<0.001$ in CORT versus vehicle; $\# p<0.05, \# \# p<0.01$ in CORT versus CORT $+\mathrm{FLX} ; \S p<0.05$ in vehicle versus $\mathrm{FLX}$.

born in the adult SVZ are mostly directed to the striatum and not to the OB (Ernst et al., 2014). Moreover, one cannot rule out the possibility that the CORT-induced hyposmia revealed in this study could be partly linked to a deleterious effect of CORT on preexisting granule and periglomerular cells in the OB. This could also be the case in human patients suffering from MDD. Finally, because glucocorticoid receptors are widely distributed in the $\mathrm{OB}$ and olfactory cortex, as in other limbic areas, notably the hippocampus, amygdala, and hypothalamus (Morimoto et al., 1996), it is possible that CORT-induced changes in overall 
cortical and limbic processing could be linked to the observed olfactory deficits.

Our results also showed that chronic CORT impairs both proliferation and survival of progenitor cells in the DG, demonstrating a role for glucocorticoids in several steps of the neurogenic process. Our data are in line with previous work reporting that ablation of the adrenal glands abolishes the stress-induced decrease in cell proliferation in the DG (Tanapat et al., 2001). Although CORT reduced cell proliferation in the DG, it did not affect cell proliferation in the $\mathrm{OE}$ nor the number of newly generated immature neurons expressing DCX in the SVZ. Our results indicate that the early steps of adult neurogenesis are differentially regulated by CORT in the DG, EO, and SVZ. However, given the decrease in the population of adult-born neurons in the $\mathrm{OB}$, it is possible that CORT treatment alters the survival rate of adult-born $\mathrm{OB}$ neurons. Interestingly, their dendritic morphology was not changed by CORT, suggesting that the surviving neurons are functionally integrated in the $\mathrm{OB}$ circuit. This hypothesis is also supported by the fact that the distribution and degree of the sensory afferents reaching the $\mathrm{OB}$, was unchanged. Concerning the adult olfactory system, the functional importance of CORT remains largely unknown. New experiments aiming to assess firing activity, dendritic dynamics, and innate responses to odorants are needed to decipher the precise role of CORT in olfactory processing and odor-driven behaviors.

\section{Chronic FLX treatment attenuates CORT-induced olfactory deficits and restores adult neurogenesis}

Our findings show that the antidepressant FLX can restore olfactory acuity and memory in CORT-treated mice. Interestingly, we also showed that FLX restores olfactory neurogenesis and facilitates the maturation of adult-born neurons in the OB and hippocampus. This proneurogenic effect could partly support the FLX-induced restoration of olfactory deficits. Similarly, recruitment of newborn neurons was recently reported to selectively restore circuits in the lesioned $\mathrm{OB}$ and resume olfaction (Lazarini et al., 2014).

FLX restored both proliferation and survival of progenitor cells in the DG, as previously reported (David et al., 2009; Rainer et al., 2012; Guilloux et al., 2013). By restoring hippocampal neurogenesis, FLX probably helps reestablish HPA axis function, resulting in functional improvement. In fact, it is argued that newly generated neurons may facilitate a dysfunctional hippocampus to restore control on stress response systems, and that the ability of FLX to restore hippocampal regulation of the HPA axis under chronic stress occurs only in the presence of an intact neurogenic niche. Further experiments aiming to selectively block either DG or SVZ neurogenesis in our model are required to disambiguate the role of each site of adult neurogenesis in the response to FLX and their associated behavioral rehabilitation.

Finally, to explain the olfactory deficits induced by FLX alone, one should keep in mind that the $\mathrm{OB}$ is one of the most densely innervated serotonergic targets of the raphe nuclei, and fibers from the latter are the densest centrifugal projections in the $\mathrm{OB}$ (Dugué and Mainen, 2009). Serotonin alters synaptic transmission and information processing in the glomerular layer of the $\mathrm{OB}$, where the density of serotonergic fibers from the raphe nuclei is maximal. As it has been indicated by early experiments, local application of serotonin receptor agonists attenuates odorevoked olfactory neuron activity (Bloom et al., 1964), whereas serotonin receptor antagonists boost glomerular activation, showing that endogenous serotonin suppresses odor input (Petzold et al., 2009). These previous studies could explain the deficits in olfactory acuity, discrimination, and long-term memory obtained by chronic FLX treatment.

Together, our findings introduce novel effects of chronic CORT and FLX on olfaction and olfactory neurogenesis in the mouse. These observations bring further insights on the poorly known biological underpinnings of anxiodepressive states and help move toward more effective therapeutic ends targeting neurogenesis, as well as useful diagnostic tools based on olfaction.

\section{References}

Alonso M, Lepousez G, Sebastien W, Bardy C, Gabellec MM, Torquet N, Lledo PM (2012) Activation of adult-born neurons facilitates learning and memory. Nat Neurosci 15:897-904. CrossRef Medline

Anacker C, Zunszain PA, Carvalho LA, Pariante CM (2011) The glucocorticoid receptor: pivot of depression and of antidepressant treatment? Psychoneuroendocrinology 36:415-425. CrossRef Medline

Belnoue L, Grosjean N, Abrous DN, Koehl M (2011) A critical time window for the recruitment of bulbar newborn neurons by olfactory discrimination learning. J Neurosci 31:1010-1016. CrossRef Medline

Bloom FE, Costa E, Salmoiraghi GC (1964) Analysis of individual rabbit olfactory bulb neuron responses to the microelectrophoresis of acetylcholine, norepinephrine and serotonin synergists and antagonists. J Pharmacol Exp Ther 146:16-23. Medline

Boldrini M, Underwood MD, Hen R, Rosoklija GB, Dwork AJ, John Mann J, Arango V (2009) Antidepressants increase neural progenitor cells in the human hippocampus. Neuropsychopharmacology 34:2376-2389. CrossRef Medline

Breton-Provencher V, Lemasson M, Peralta MR 3rd, Saghatelyan A (2009) Interneurons produced in adulthood are required for the normal functioning of the olfactory bulb network and for the execution of selected olfactory behaviors. J Neurosci 29:15245-15257. CrossRef Medline

Campbell S, Marriott M, Nahmias C, MacQueen GM (2004) Lower hippocampal volume in patients suffering from depression: a meta-analysis. Am J Psychiatry 161:598-607. CrossRef Medline

Chen H, Pandey GN, Dwivedi Y (2006) Hippocampal cell proliferation regulation by repeated stress and antidepressants. Neuroreport 17:863-867. CrossRef Medline

Croy I, Negoias S, Symmank A, Schellong J, Joraschky P, Hummel T (2013) Reduced olfactory bulb volume in adults with a history of childhood maltreatment.Chem Senses 38:679-684. CrossRef

Cryan JF, Mombereau C, Vassout A (2005) The tail suspension test as a model for assessing antidepressant activity: review of pharmacological and genetic studies in mice. Neurosci Biobehav Rev 29:571-625. CrossRef Medline

David DJ, Samuels BA, Rainer Q, Wang JW, Marsteller D, Mendez I, Drew M, Craig DA, Guiard BP, Guilloux JP, Artymyshyn RP, Gardier AM, Gerald C, Antonijevic IA, Leonardo ED, Hen R (2009) Neurogenesisdependent and -independent effects of fluoxetine in an animal model of anxiety/depression. Neuron 62:479-493. CrossRef Medline

de Chaumont F, Dallongeville S, Chenouard N, Hervé N, Pop S, Provoost T, Meas-Yedid V, Pankajakshan P, Lecomte T, Le Montagner Y, Lagache T, Dufour A, Olivo-Marin JC (2012) Icy: an open bioimage informatics platform for extended reproducible research. Nat Methods 9:690-696. CrossRef Medline

Dranovsky A, Hen R (2006) Hippocampal neurogenesis: regulation by stress and antidepressants. Biol Psychiatry 59:1136-1143. CrossRef Medline

Dugué GP, Mainen ZF (2009) How serotonin gates olfactory information flow. Nat Neurosci 12:673-675. CrossRef Medline

Ernst A, Alkass K, Bernard S, Salehpour M, Perl S, Tisdale J, Possnert G, Druid H, Frisén J (2014) Neurogenesis in the striatum of the adult human brain. Cell 156:1072-1083. CrossRef Medline

Garza JC, Guo M, Zhang W, Lu XY (2012) Leptin restores adult hippocampal neurogenesis in a chronic unpredictable stress model of depression and reverses glucocorticoid-induced inhibition of GSK- $3 \beta / \beta$-catenin signaling. Mol Psychiatry 17:790-808. CrossRef Medline

Glinka ME, Samuels BA, Diodato A, Teillon J, Feng Mei D, Shykind BM, Hen R, Fleischmann A (2012) Olfactory deficits cause anxiety-like behaviors in mice. J Neurosci 32:6718-6725. CrossRef Medline

Guilloux JP, Mendez-David I, Pehrson A, Guiard BP, Repérant C, Orvoën S, Gardier AM, Hen R, Ebert B, Miller S, Sanchez C, David DJ (2013) An- 
tidepressant and anxiolytic potential of the multimodal antidepressant vortioxetine (Lu AA21004) assessed by behavioral and neurogenesis outcomes in mice. Neuropharmacology 73:147-159. CrossRef Medline

Harris BN, Perea-Rodriguez JP, Saltzman W (2011) Acute effects of corticosterone injection on paternal behavior in California mouse (Peromyscus californicus) fathers. Horm Behav 60:666-675. CrossRef Medline

Hitoshi S, Maruta N, Higashi M, Kumar A, Kato N, Ikenaka K (2007) Antidepressant drugs reverse the loss of adult neural stem cells following chronic stress. J Neurosci Res 85:3574-3585. CrossRef Medline

Krishnan V, Nestler EJ (2008) The molecular neurobiology of depression. Nature 455:894-902. CrossRef Medline

Lau BW, Yau SY, Lee TM, Ching YP, Tang SW, So KF (2011) Effect of corticosterone and paroxetine on masculine mating behavior: possible involvement of neurogenesis. J Sex Med 8:1390-1403. CrossRef Medline

Lazarini F, Lledo PM (2011) Is adult neurogenesis essential for olfaction? Trends Neurosci 34:20-30. CrossRef Medline

Lazarini F, Gabellec MM, Torquet N, Lledo PM (2012) Early activation of microglia triggers long-lasting impairment of adult neurogenesis in the olfactory bulb. J Neurosci 32:3652-3664. CrossRef Medline

Lazarini F, Gabellec MM, Moigneu C, de Chaumont F, Olivo-Marin JC, Lledo PM (2014) Adult neurogenesis restores dopaminergic neuronal loss in the olfactory bulb.J Neurosci 34:14430-14442. CrossRef

Lehmann ML, Brachman RA, Martinowich K, Schloesser RJ, Herkenham M (2013) Glucocorticoids orchestrate divergent effects on mood through adult neurogenesis. J Neurosci 33:2961-2972. CrossRef Medline

Linster C, Johnson BA, Morse A, Yue E, Leon M (2002) Spontaneous vs reinforced olfactory discriminations. J Neurosci 22:6842-6845. Medline

Lledo PM, Alonso M, Grubb MS (2006) Adult neurogenesis and functional plasticity in neuronal circuits. Nat Rev Neurosci 7:179-193. CrossRef Medline

Mendez-David I, David DJ, Darcet F, Wu MV, Kerdine-Römer S, Gardier AM, Hen R (2014) Rapid anxiolytic effects of a 5-HT4 receptor agonist are mediated by a neurogenesis-independent mechanism. Neuropsychopharmacology 39:1366-1378. CrossRef Medline

Mirescu C, Gould E (2006) Stress and adult neurogenesis. Hippocampus 16:233-238. CrossRef Medline

Moreno MM, Linster C, Escanilla O, Sacquet J, Didier A, Mandairon N (2009) Olfactory perceptual learning requires adult neurogenesis. Proc Natl Acad Sci U S A 106:17980-17985. CrossRef Medline

Morimoto M, Morita N, Ozawa H, Yokoyama K, Kawata M (1996) Distribution of glucocorticoid receptor immunoreactivity and mRNA in the rat brain: an immunohistochemical and in situ hybridization study. Neurosci Res 26:235-269. CrossRef Medline

Mouret A, Lepousez G, Gras J, Gabellec MM, Lledo PM (2009) Turnover of newborn olfactory bulb neurons optimizes olfaction.J Neurosci 29: 12302-12314. CrossRef

Murray F, Smith DW, Hutson PH (2008) Chronic low dose corticosterone exposure decreased hippocampal cell proliferation, volume and induced anxiety and depression like behaviors in mice. Eur J Pharmacol 583:115127. CrossRef Medline

Negoias S, Croy I, Gerber J, Puschmann S, Petrowski K, Joraschky P, Hummel $\mathrm{T}$ (2010) Reduced olfactory bulb volume and olfactory sensitivity in patients with acute major depression. Neuroscience 169:415-421. CrossRef Medline

Olivo-Marin JC (2002) Extraction of spots in biological images using multiscale products. Pattern Recognit 35:1986-1996.

Pallotto M, Nissant A, Fritschy JM, Rudolph U, Sassoè-Pognetto M, Panzanelli P, Lledo PM (2012) Early formation of GABAergic synapses governs the development of adult-born neurons in the olfactory bulb. J Neurosci 32:9103-9115. CrossRef Medline

Pause BM, Miranda A, Göder R, Aldenhoff JB, Ferstl R (2001) Reduced olfactory performance in patients with major depression. J Psychiatr Res 35:271-277. CrossRef Medline

Petzold GC, Hagiwara A, Murthy VN (2009) Serotonergic modulation of odor input to the mammalian olfactory bulb. Nat Neurosci 12:784-791. CrossRef Medline

Rainer Q, Xia L, Guilloux JP, Gabriel C, Mocaër E, Hen R, Enhamre E, Gardier AM, David DJ (2012) Beneficial behavioral and neurogenic effects of agomelatine in a model of depression/anxiety. Int J Neuropsychopharmacol 15:321-335. CrossRef Medline

Sahay A, Hen R (2007) Adult hippocampal neurogenesis in depression. Nat Neurosci 10:1110-1115. CrossRef Medline

Sakamoto M, Ieki N, Miyoshi G, Mochimaru D, Miyachi H, Imura T, Yamaguchi M, Fishell G, Mori K, Kageyama R, Imayoshi I (2014) Continuous postnatal neurogenesis contributes to formation of the olfactory bulb neural circuits and flexible olfactory associative learning. J Neurosci 34: 5788-5799. CrossRef Medline

Santarelli L, Saxe M, Gross C, Surget A, Battaglia F, Dulawa S, Weisstaub N, Lee J, Duman R, Arancio O, Belzung C, Hen R (2003) Requirement of hippocampal neurogenesis for the behavioral effects of antidepressants. Science 301:805-809. CrossRef Medline

Selye H (1950) Stress and the general adaptation syndrome. Br Med J 1:1383-1392. CrossRef Medline

Snyder JS, Soumier A, Brewer M, Pickel J, Cameron HA (2011) Adult hippocampal neurogenesis buffers stress responses and depressive behavior. Nature 476:458-461. CrossRef Medline

Surget A, Saxe M, Leman S, Ibarguen-Vargas Y, Chalon S, Griebel G, Hen R, Belzung C (2008) Drug-dependent requirement of hippocampal neurogenesis in a model of depression and of antidepressant reversal. Biol Psychiatry 64:293-301. CrossRef Medline

Tanapat P, Hastings NB, Rydel TA, Galea LA, Gould E (2001) Exposure to fox odor inhibits cell proliferation in the hippocampus of adult rats via an adrenal hormone-dependent mechanism. J Comp Neurol 437:496-504. CrossRef Medline

Wren A, van Riezen H, Rigter H (1977) A new animal model for the prediction of antidepressant activity. Pharmakopsychiatr Neuropsychopharmakol 10:96-100. CrossRef Medline 